NASA Technical Memorandum 110337

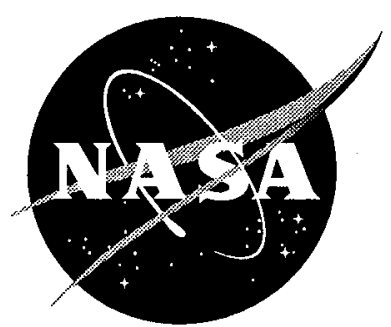

\title{
External Boundary Conditions for Three-Dimensional Problems of Computational Aerodynamics
}

Semyon V. Tsynkov

Langley Research Center, Hampton, Virginia

March 1997

National Aeronautics and

Space Administration

Langley Research Center

Hampton, Virginia 23681-0001 


\title{
EXTERNAL BOUNDARY CONDITIONS FOR THREE-DIMENSIONAL PROBLEMS OF COMPUTATIONAL AERODYNAMICS*
}

\author{
SEMYON V. TSYNKOV ${ }^{\dagger}$
}

\begin{abstract}
We consider an unbounded steady-state flow of viscous fluid over a three-dimensional finite body or configuration of bodies. For the purpose of solving this flow problem numerically, we discretize the governing equations (Navier-Stokes) on a finite-difference grid. The grid obviously cannot stretch from the body up to infinity, because the number of the discrete variables in that case would not be finite. Therefore, prior to the discretization we truncate the original unbounded flow domain by introducing some artificial computational boundary at a finite distance of the body. Typically, the artificial boundary is introduced in a natural way as the external boundary of the domain covered by the grid.

The flow problem formulated only on the finite computational domain rather than on the original infinite domain is clearly subdefinite unless some artificial boundary conditions ( $A B C$ 's) are specified at the external computational boundary. Similarly, the discretized flow problem is subdefinite (i.e., lacks equations with respect to unknowns) unless a special closing procedure is implemented at this artificial boundary. The closing procedure in the discrete case is called the ABC's as well.

In this paper, we present an innovative approach to constructing highly accurate ABC's for three-dimensional flow computations. The approach extends our previous technique developed for the two-dimensional case; it employs the finite-difference counterparts to Calderon's pseudodifferential boundary projections calculated in the framework of the difference potentials method (DPM) by Ryaben'kii. The resulting ABC's appear spatially nonlocal but particularly easy to implement along with the existing solvers.

The new boundary conditions have been successfully combined with the NASA-developed production code TLNS3D and used for the analysis of wing-shaped configurations in subsonic (including incompressible limit) and transonic flow regimes. As demonstrated by the computational experiments and comparisons with the standard (local) methods, the DPM-based ABC's allow one to greatly reduce the size of the computational domain while still maintaining high accuracy of the numerical solution. Moreover, they may provide for a noticeable increase of the convergence rate of multigrid iterations.
\end{abstract}

Key words. External flows, infinite-domain problems, artificial boundary conditions, far-field linearization, boundary projections, generalized potentials, difference potentials method, auxiliary problem, separation of variables.

AMS subject classifications. 65N99, 76M25

\section{Introduction.}

1.1. Preliminaries. A standard approach to solving infinite-domain boundary-value problems on the computer involves truncation as a first step, prior to the discretization of the continuous problem and the solution of the resulting discrete system. The truncated problem in both continuous and discrete formulations is clearly subdefinite unless supplemented by the proper closing procedure at the outer computational boundary. The latter boundary is often called artificial emphasizing the fact that it originates from the numerical limitations (the discrete system should contain no more than a finite number of variables) rather than original formulation. Typically, the artificial boundary is introduced as an external boundary of the finite computational domain (i.e., the domain covered by the grid, on which the original system is discretized). The corresponding closing procedure at the outer boundary is called the artificial boundary conditions ( $A B C$ 's).

In the ideal case, the $A B C$ 's would be specified so that the solution on the truncated domain coincides with the corresponding fragment of the original infinite-domain solution. However, in spite of the fact that different ABC's methodologies have been studied extensively over the recent two decades, the construction of such ideal (i.e., exact) $A B C$ 's that would at the same time be computationally inexpensive, easy to implement, and geometrically universal, still remains a fairly

${ }^{*}$ This paper was prepared while the author held a National Research Council Resident Research Associateship at NASA Langley Research Center.

${ }^{\dagger}$ Mail Stop 128, NASA Langley Research Center, Hampton, VA 23681-0001, U.S.A. Phone: (1-757)864-2150; Fax: (1-757)864-8816; E-mail: s.v.tsynkov@larc.nasa.gov; URL: http://fmad-www.larc.nasa.gov:80/ tsynkov/. 
remote possibility. The primary reason for that is that the exact $\mathrm{ABC}$ 's are typically nonlocal, for steady-state problems in space and for time-dependent problems also in time. The exceptions are rare and, as a rule, restricted to the model one-dimensional examples. From the viewpoint of computing, nonlocality may imply cumbersomeness and high cost. Moreover, as the standard apparatus for deriving the exact ABC's involves integral transforms (along the boundary) and pseudodifferential operators, such boundary conditions can be obtained easily only for the boundaries of regular shape.

On the other hand, highly accurate ABC's are most demanded in many areas of scientific computing because as shown by the different authors both theoretically and computationally, the overall accuracy and performance of numerical algorithms, as well as interpretation of the numerical results, strongly depend on the proper treatment of external boundaries. The possible range of applications for different ABC's techniques is broad. Aerodynamics, in which external problems represent a wide class of important applications, especially when it comes to the analysis of threedimensional configurations, constitutes a fraction of this range. Besides the hydro- and aerodynamic problems (external flows, duct flows, boundary layers, free surfaces, etc.), the entire range includes the flows in porous media, filtration, magneto-hydrodynamic flows, plasma (e.g., solar wind), the problems of solid mechanics (in particular, elasticity and aeroelasticity), and the problems of wave propagation (electromagnetic, acoustic, seismic), just to name a few.

As mentioned above, the other usual requirements of ABC's, besides minimization of the error associated with the domain truncation, are low computational cost, geometric universality (i.e., applicability to a variety of irregular boundaries often encountered in real-life settings), and implementation without difficulties, in particular, readiness in combining the ABC's with the existing (interior) solvers. The requirements of this group are typically met by many approximate local methods that are considered an alternative to the exact ABC's as the latter are not attainable routinely. However, the basic trend in terms of accuracy remains the following: higher accuracy for the boundary procedure requires more of the nonlocal nature of exact ABC's to be somehow taken into account.

In fact, almost any numerical algorithm for setting the ABC's can be thought of as a compromise between the two foregoing groups of requirements that in a certain sense contradict one another. Shifting the balance towards locality and practical efficacy often implies insufficient accuracy; shifting it to the other side, towards highly accurate nonlocal techniques, may often yield cumbersome and all but impractical algorithms. It is not surprising, therefore, that the treatment of external boundaries in modern production computations typically follows the first, local, path. In computational fluid dynamics (CFD), for example, only a few ABC's methodologies out of the wide variety proposed to date can be regarded as the commonly used tools. All of them are either based on the essential model simplifications, e.g., local quasi-one-dimensional treatment in the vicinity of the artificial boundary, or obtained as a localization of some nonlocal ABC's. To meet the overall accuracy requirements when using such simple boundary procedures, one often has to choose the excessively large computational domains.

A survey of methods for setting the ABC's in different areas of scientific computing can be found in our work [1], as well as in the comprehensive reviews by Givoli [2,3]. These surveys give a. comparative assessment of advantages and disadvantages of various global and local techniques, and also point out the relations between the global and local methods.

1.2. Methods and Objectives. This paper continues our work on constructing the ABC's that would combine the advantages relevant to both local and nonlocal approaches. The specific area of applications that we are looking at is steady-state external viscous flows.

Previously, we have developed and implemented in practice the highly accurate ABC's for twodimensional case (plane geometry). Our approach is based on usage of the Calderon generalized potentials and pseudodifferential boundary projections [4] (see also work by Seeley [5]). The po- 
tentials and projections are actually employed in the modified form proposed by Ryaben'kii; the corresponding numerical technique used for calculating the potentials and projections is known as the difference potentials method (DPM), see work by Ryaben'kii $[6,7,8]$ and also the description of the method in the book by Mikhlin, et al. [9]. The resulting DPM-based boundary conditions appear global. As, however, will be seen, one of the principal advantages that we gain using the DPM is that the method allows us to simultaneously meet the high accuracy standards and the requirements of geometric universality and easiness in implementation.

The two-dimensional DPM-based ABC's have been used along with the multigrid Navier-Stokes code FLOMG by Swanson and Turkel $[10,11,12]$. In spite of their nonlocal nature, the new boundary conditions readily apply to the boundaries of irregular shape and appear very easy to incorporate into the existing solver. In our computations, the DPM-based ABC's have explicitly outperformed the standard local methods from the standpoints of accuracy, convergence rate, and robustness. The investigated regimes range from the very low (incompressible limit) to transonic Mach numbers and encompass both laminar and turbulent flows.

The aforementioned two-dimensional constructions and the corresponding numerical results have been reported in a series of papers. In work [13], we describe the foundations of the DPMbased approach to setting the ABC's for computation of two-dimensional external viscous flows (Navier-Stokes equations). In work [14], we implement this approach along with the code FLOMG and present some numerical results for subsonic and transonic laminar flows over single-element airfoils. In work [15], we show the results of subsequent numerical experiments and propose an approximate treatment of turbulence in the far field. Our work [16] delineates the algorithm for solving one-dimensional systems of ordinary difference equations that arise when calculating the generalized difference potentials. In work [17], we extend the area of applications for the DPM-based ABC's by analyzing two-dimensional flows that oscillate in time; we also provide some solvability results for the linearized thin-layer equations used for constructing the ABC's. In work [18], we present a general survey of the DPM-based methodology as applied to solving external problems in CFD, including parallel implementation of the algorithm, combined implementation of nonlocal ABC's with multigrid, and entry-wise interpolation of the matrices of boundary operators with respect to the Mach number and the angle of attack. Additionally, in [18] one can find some new theoretical results on the computation of generalized potentials, the construction of ABC's based on the direct implementation of boundary projections (thin-layer equations), and some numerical results for various airfoil flows: laminar and turbulent, transonic and subsonic, including very low Mach numbers.

The next natural objective after constructing the two-dimensional algorithm is the analysis of three-dimensional steady-state flows. This case is undoubtedly the one most demanded by the current practice in CFD. In work $[19,20]$, we outline the basic elements of the DPM-based ABC's for steady-state viscous flows around the wing-shaped configurations and show some preliminary numerical results for the subsonic regime. The numerical results of work [20] are obtained with the NASA-developed production code TLNS3D by Vatsa, et al. [21]. In work [22] we further develop the three-dimensional DPM-based algorithm and present the computational results for transonic flows. In all cases (see $[20,22]$ ), the DPM-based ABC's allow one to greatly reduce the size of the computational domain (compared to the standard local boundary conditions) while still maintaining high accuracy of the numerical solution. This actually means the overall increase of accuracy due to the improved treatment of the artificial boundary; it also implies the substantial economy of the computer resources. Moreover, the DPM-based ABC's may provide for a noticeable speedup (up to a factor of three) of the convergence of multigrid iterations.

Below, we for the first time systematically describe the three-dimensional DPM-based ABC's for calculating viscous flows around the wings. We address the theoretical foundations of the approach, present the numerical algorithm with a fair amount of details, and demonstrate the 
computational results for the different flow regimes, including the low speed flow and the flow with the developed separation. The numerical results for the DPM-based ABC's are compared with those obtained with the standard local method.

The material in the paper is prepared as follows. In Section 2, we formulate the problem, describe the specific geometric setting for three space dimensions, provide the foundations for the DPM-based ABC's on the continuous level, and then implement the new algorithm in the finitedifference framework. In Section 3, we first briefly summarize the results of our previous numerical experiments in two space dimensions and then report on the recent three-dimensional computations for various flow regimes. Section 4 contains the conclusions.

\section{External Flow.}

2.1. Formulation of the Problem. We consider an unbounded steady-state flow of viscous fluid past a three-dimensional wing. The flow is uniform at infinity. We consider both incompressible and compressible formulations, in the latter case we assume that the fluid (gas) is thermodynamically perfect and that the free stream is subsonic. Moreover, as the fluid is viscous and the size of the immersed body (wing) is finite, the flow limit at infinity is a free stream.

Generally, the near-field flow is governed by the full Navier-Stokes equations. However, in many cases (including those studied in this paper, see Section 2.2) the full system can be simplified and reduced to the so-called thin-layer equations [23], which do not contain streamwise viscous derivatives. In particular, this simplification is done in the code TLNS3D that we are using for our numerical tests (Section 3). Moreover, for the most interesting case of turbulent flows the nearfield numerical algorithm should also involve some turbulence model, we comment on this issue in Section 3, which is devoted to numerics.

2.1.1. Linearization. In the far field (i.e., far enough from the finite immersed body), the perturbations of the flow induced by the immersed body are small and we therefore linearize the governing thin-layer equations against the constant free-stream background. Introducing the Cartesian coordinates $(x, y, z)$ and assuming (without loss of generality) that the free stream is aligned with the positive $x$ direction, we can write the dimensionless linearized equations as

$$
\boldsymbol{L} \boldsymbol{u} \equiv \boldsymbol{C} \frac{\partial \boldsymbol{u}}{\partial x}+\boldsymbol{D} \frac{\partial \boldsymbol{u}}{\partial y}+\boldsymbol{E} \frac{\partial \boldsymbol{u}}{\partial z}+\boldsymbol{F} \frac{\partial^{2} \boldsymbol{u}}{\partial y^{2}}+\boldsymbol{H} \frac{\partial^{2} \boldsymbol{u}}{\partial z^{2}}+\boldsymbol{J} \frac{\partial^{2} \boldsymbol{u}}{\partial y \partial z}=\mathbf{o}
$$

where for the incompressible case

$$
\begin{gathered}
(2.1 \mathrm{~b}) \boldsymbol{u}=\left[\begin{array}{c}
p \\
u \\
v \\
w
\end{array}\right], \boldsymbol{C}=\left[\begin{array}{llll}
0 & 1 & 0 & 0 \\
1 & 1 & 0 & 0 \\
0 & 0 & 1 & 0 \\
0 & 0 & 0 & 1
\end{array}\right], \boldsymbol{D}=\left[\begin{array}{llll}
0 & 0 & 1 & 0 \\
0 & 0 & 0 & 0 \\
1 & 0 & 0 & 0 \\
0 & 0 & 0 & 0
\end{array}\right], \quad \boldsymbol{E}=\left[\begin{array}{llll}
0 & 0 & 0 & 1 \\
0 & 0 & 0 & 0 \\
0 & 0 & 0 & 0 \\
1 & 0 & 0 & 0
\end{array}\right], \\
\boldsymbol{F}=\boldsymbol{H}=-\frac{1}{R e}\left[\begin{array}{llll}
0 & 0 & 0 & 0 \\
0 & 1 & 0 & 0 \\
0 & 0 & 1 & 0 \\
0 & 0 & 0 & 1
\end{array}\right], \quad \boldsymbol{J}=\mathbf{0},
\end{gathered}
$$

and for the compressible case 


$$
\begin{aligned}
& \boldsymbol{u}=\left[\begin{array}{c}
\rho \\
u \\
v \\
w \\
p
\end{array}\right], \quad \boldsymbol{C}=\left[\begin{array}{ccccc}
1 & 1 & 0 & 0 & 0 \\
0 & 1 & 0 & 0 & 0 \\
0 & 0 & 1 & 0 & 0 \\
0 & 0 & 0 & 1 & 0 \\
0 & M_{0}^{-2} & 0 & 0 & 1
\end{array}\right], \quad \boldsymbol{D}=\left[\begin{array}{ccccc}
0 & 0 & 1 & 0 & 0 \\
0 & 0 & 0 & 0 & 0 \\
0 & 0 & 0 & 0 & 1 \\
0 & 0 & 0 & 0 & 0 \\
0 & 0 & M_{0}^{-2} & 0 & 0
\end{array}\right] \\
& \boldsymbol{E}=\left[\begin{array}{ccccc}
0 & 0 & 0 & 1 & 0 \\
0 & 0 & 0 & 0 & 0 \\
0 & 0 & 0 & 0 & 0 \\
0 & 0 & 0 & 0 & 1 \\
0 & 0 & 0 & M_{0}^{-2} & 0
\end{array}\right], \quad \boldsymbol{F}=-\frac{1}{R e}\left[\begin{array}{ccccc}
0 & 0 & 0 & 0 & 0 \\
0 & 1 & 0 & 0 & 0 \\
0 & 0 & 4 / 3 & 0 & 0 \\
0 & 0 & 0 & 1 & 1 \\
P r^{-1} M_{0}^{-2} & 0 & 0 & 0 & \gamma P r^{-1}
\end{array}\right], \\
& \boldsymbol{H}=-\frac{1}{R e}\left[\begin{array}{ccccc}
0 & 0 & 0 & 0 & 0 \\
0 & 1 & 0 & 0 & 0 \\
0 & 0 & 1 & 0 & 0 \\
0 & 0 & 0 & 4 / 3 & 1 \\
P r^{-1} M_{0}^{-2} & 0 & 0 & 0 & \gamma P r^{-1}
\end{array}\right], \quad \boldsymbol{J}=-\frac{1}{R e}\left[\begin{array}{ccccc}
0 & 0 & 0 & 0 & 0 \\
0 & 0 & 0 & 0 & 0 \\
0 & 0 & 0 & 1 / 3 & 0 \\
0 & 0 & 1 / 3 & 0 & 0 \\
0 & 0 & 0 & 0 & 0
\end{array}\right] .
\end{aligned}
$$

Here, $u, v, w$, and $p$ are the perturbations of the Cartesian velocity components and pressure, respectively, and $\rho$ is the perturbation of density for the compressible case.

The derivation of equations (2.1) involves two consecutive steps. First, we introduce the dimensionless quantities for the original thin-layer system. To do that, in both incompressible and compressible case we scale the velocity projections by the dimensional free-stream value of the $x$-component $u_{0}$. (As mentioned above, the free-stream $y$ and $z$ velocity components are zero: $v_{0}=0, w_{0}=0$.) Moreover, for the incompressible flow we scale the original pressure by $u_{0}{ }^{2}$ and for the compressible flow the pressure is scaled by $\rho_{0} u_{0}{ }^{2}$, the internal energy $\epsilon$ is scaled by $u_{0}{ }^{2}$ and the viscosity $\mu$ is scaled by $\mu_{0}$. (Everywhere above, the subscript " 0 " denotes the corresponding "full," i.e., "nonlinear," dimensional value.) Finally, we scale the coordinates $x, y$, and $z$ by the characteristic length $L$, for example, it may be the root chord or the semi-span of the wing (see Section 2.2). For the compressible flow, we also have to use the equation of state of the perfect gas to eliminate the internal energy from the original system.

After the nondimensionalization, we represent each quantity (velocities and pressure for the incompressible case and velocities, pressure, and density for the compressible case) as a sum of the constant background value (free stream) and the small perturbation and retain in the equations only the first-order terms with respect to the perturbations. (Note, in the incompressible case only the gradient of the pressure is involved in the original system, therefore the actual value of the background constant for the pressure does not matter.) In so doing, we arrive at equations (2.1a), (2.1b) for the incompressible flow and equations (2.1a), (2.1c) for the compressible flow. Both in (2.1b) and (2.1c) $R e$ is the Reynolds number (in the turbulent case, it is an effective far-field value, see [15]); in addition, for the compressible flow (see (2.1c)) $M_{0}=u_{0} /\left(\gamma p_{0} / \rho_{0}\right)^{1 / 2}$ is the free-stream Mach number (always $M_{0}<1$ ), $P r$ is the Prandtl number, and $\gamma$ is the ratio of specific heats.

Note, for the incompressible case it is clear that the differential equations for the small perturbations are linear. For the compressible case, however, this fact may require some additional justification, see Section 2.1.2.

The system (2.1) describes the flow in the far field. In both incompressible and compressible cases, it is supplemented by the boundary condition

$$
\boldsymbol{u} \longrightarrow \mathbf{o}, \quad \text { as } \quad r \equiv\left(x^{2}+y^{2}+z^{2}\right)^{1 / 2} \longrightarrow+\infty
$$


which means that all the perturbations vanish at infinity, or equivalently, the flow approaches the free stream.

Let us mention, that the matrices $\boldsymbol{C}, \boldsymbol{D}, \boldsymbol{E}, \boldsymbol{F}, \boldsymbol{H}$ of (2.1b) are symmetric, whereas the matrices $\boldsymbol{C}, \boldsymbol{D}, \boldsymbol{E}, \boldsymbol{F}, \boldsymbol{H}$ of (2.1c) are not. As the symmetric form of the matrices may sometimes be more convenient for the analysis and also more suitable for the numerical calculations (especially when the Mach number $M_{0}$ is low), one can use the transformation proposed by Abarbanel and Gottlieb in work [24] to simultaneously reduce $\boldsymbol{C}, \boldsymbol{D}, \boldsymbol{E}, \boldsymbol{F}, \boldsymbol{H}$ of (2.1c) to the symmetric (and some of the matrices to the diagonal) form. Specifically, introducing the non-degenerate matrix $S$,

$$
S=\left[\begin{array}{ccccc}
\sqrt{\gamma} M_{0} & 0 & 0 & 0 & 0 \\
0 & 1 & 0 & 0 & 0 \\
0 & 0 & 1 & 0 & 0 \\
0 & 0 & 0 & 1 & 0 \\
\left(\sqrt{\gamma} M_{0}\right)^{-1} & 0 & 0 & 0 & \sqrt{\gamma-1}\left(\sqrt{\gamma} M_{0}\right)^{-1}
\end{array}\right]
$$

with the inverse

$$
S^{-1}=\left[\begin{array}{ccccc}
\left(\sqrt{\gamma} M_{0}\right)^{-1} & 0 & 0 & 0 & 0 \\
0 & 1 & 0 & 0 & 0 \\
0 & 0 & 1 & 0 & 0 \\
0 & 0 & 0 & 1 & 0 \\
-\left(\sqrt{\gamma(\gamma-1)} M_{0}\right)^{-1} & 0 & 0 & 0 & \sqrt{\gamma-1}\left(\sqrt{\gamma} M_{0}\right)^{-1}
\end{array}\right]
$$

we have instead of $(2.1 \mathrm{c})$

$$
\begin{aligned}
& \tilde{\boldsymbol{C}}=\boldsymbol{S}^{-1} \boldsymbol{C} \boldsymbol{S}=\left[\begin{array}{ccccc}
1 & \left(\sqrt{\gamma} M_{0}\right)^{-1} & 0 & 0 & 0 \\
\left(\sqrt{\gamma} M_{0}\right)^{-1} & 1 & 0 & 0 & \sqrt{\gamma-1}\left(\sqrt{\gamma} M_{0}\right)^{-1} \\
0 & 0 & 1 & 0 & 0 \\
0 & 0 & 0 & 1 & 0 \\
0 & \sqrt{\gamma-1}\left(\sqrt{\gamma} M_{0}\right)^{-1} & 0 & 0 & 1
\end{array}\right] \\
& \tilde{\boldsymbol{D}}=\boldsymbol{S}^{-1} \boldsymbol{D} \boldsymbol{S}=\left[\begin{array}{ccccc}
0 & 0 & \left(\sqrt{\gamma} M_{0}\right)^{-1} & 0 & 0 \\
0 & 0 & 0 & 0 & 0 \\
\left(\sqrt{\gamma} M_{0}\right)^{-1} & 0 & 0 & 0 & \sqrt{\gamma-1}\left(\sqrt{\gamma} M_{0}\right)^{-1} \\
0 & 0 & 0 & 0 & 0 \\
0 & 0 & \sqrt{\gamma-1}\left(\sqrt{\gamma} M_{0}\right)^{-1} & 0 & 0
\end{array}\right] \\
& \tilde{E}=\boldsymbol{S}^{-1} \boldsymbol{E} \boldsymbol{S}=\left[\begin{array}{ccccc}
0 & 0 & 0 & \left(\sqrt{\gamma} M_{0}\right)^{-1} & 0 \\
0 & 0 & 0 & 0 & 0 \\
0 & 0 & 0 & 0 & 0 \\
\left(\sqrt{\gamma} M_{0}\right)^{-1} & 0 & 0 & 0 & \sqrt{\gamma-1}\left(\sqrt{\gamma} M_{0}\right)^{-1} \\
0 & 0 & 0 & \sqrt{\gamma-1}\left(\sqrt{\gamma} M_{0}\right)^{-1} & 0
\end{array}\right] \\
& \tilde{\boldsymbol{F}}=\boldsymbol{S}^{-1} \boldsymbol{F} \boldsymbol{S}=\left[\begin{array}{ccccc}
0 & 0 & 0 & 0 & 0 \\
0 & 1 & 0 & 0 & 0 \\
0 & 0 & 4 / 3 & 0 & 0 \\
0 & 0 & 0 & 1 & 1 \\
0 & 0 & 0 & 0 & \gamma \operatorname{Pr}^{-1}
\end{array}\right], \quad \tilde{H}=\boldsymbol{S}^{-1} \boldsymbol{H} \boldsymbol{S}=\left[\begin{array}{ccccc}
0 & 0 & 0 & 0 & 0 \\
0 & 1 & 0 & 0 & 0 \\
0 & 0 & 1 & 0 & 0 \\
0 & 0 & 0 & 4 / 3 & 1 \\
0 & 0 & 0 & 0 & \gamma P r^{-1}
\end{array}\right] \text {, } \\
& \tilde{J}=S^{-1} J S=J .
\end{aligned}
$$


The symmetric form $\tilde{\boldsymbol{C}}, \tilde{\boldsymbol{D}}, \tilde{\boldsymbol{E}}, \tilde{\boldsymbol{F}}, \tilde{\boldsymbol{H}}, \tilde{\boldsymbol{J}}$ (see (2.4)) of the system matrices appears useful when analyzing the incompressible limit $M_{0} \longrightarrow+0$. A detailed study of the compressible Euler and Navier-Stokes equations as the Mach number approaches zero can be found in work [25], as well as in work $[26,27]$.

2.1.2. Asymptotic Methods - Linear vs. Nonlinear. In connection with the linearized model proposed above for the far field, especially as it regards the compressible case, we will discuss here one group of the ABC's methods that are widely used in computations. These methods employ some asymptotic form of the far-field solution for closing the system of equations to be solved inside the computational domain. Typically, this approximate asymptotic form can be obtained as a few leading terms of the series (or asymptotic series) that represents the solution in the far field. The corresponding $\mathrm{ABC}$ 's most often appear local.

The idea of the this type was employed, for example, by Sa and Chang in work [28] to set the $A B C$ 's for vorticity when integrating the incompressible Navier-Stokes equations around a cylinder. Burkhart in [29] and Burkhart et al. in [30] derive an asymptotic expansion for the finitedifference fundamental solution of the three-dimensional Laplace operator on a Cartesian grid and then use a few leading terms of this expansion to set the ABC's for an external flow problem that is solved within the full-potential framework. Wubs, Boerstoel, and Van der Wees in [31] use a Fourier representation of the far-field solution to the two-dimensional Laplace equation to calculate a. potential flow around an airfoil. The ABC's [31] are again derived from the first few leading terms of the expansion; as the artificial boundary approaches the airfoil, more terms are required to maintain the accuracy. Finally, the so-called point-vortex model, which has been proposed by Thomas and Salas in an earlier work [32] and which is extensively used in the today's CFD is also based on the idea of asymptotics. Specifically, the first leading term of the far-field expansion for the linearized flow potential is used to calculate the velocity projections at the external boundary when computing the two-dimensional compressible flows. This leading term is proportional to the circulation of the flow.

The asymptotic methods may often require the explicit knowledge of the coefficients that multiply the corresponding terms in the expansion (the ones that are used in the far-field representation). In CFD, these coefficients are typically obtained through the boundary conditions on the surface of the immersed body. For example, the value of circulation for the point-vortex model [32] is proportional to the lift, which is calculated by integrating the pressure along the surface. There is also another way of using the asymptotics for setting the far-field ABC's. It has been proposed by Bayliss and Turkel in work [33, 34,35] and by Bayliss, Gunzburger, and Turkel in work [36] and does not require the explicit knowledge of the coefficients. Instead, the authors of $[33,34,35,36]$ develop a set of special local differential relations that identically cancel out the prescribed number of leading terms in the corresponding series; these relations can obviously serve as the ABC's. However, they are typically of a high order even if the order of the original equation (system) is low.

As a rule, the asymptotic ABC's methods are derived on the basis of the linear (or linearized) equations (apparently because it is easier to study the convergence of the corresponding series). In certain cases, however, one takes into account the nonlinear corrections as well. For example, when analyzing the transonic limit $M_{0} \longrightarrow 1$ for the small perturbations of the velocity potential in the flow of compressible gas, some second-order terms should formally be retained in the differential equation along with the first-order terms (see, e.g., the book by Cole and Cook [37]). This leads to the nonlinear Kármán-Guderley equation rather than to the linear Prandtl-Glauert equation (the latter is valid for smaller $M_{0}$ ). For two-dimensional external flows (e.g., flows around airfoils) described by the Kármán-Guderley equation, it turns out that the nonlinear corrections to the leading linear lift-based term $\sim-\Gamma \theta / 2 \pi$ in the far-field expansion of the potential ( $\Gamma$ is the flow circulation, $\theta$ is the polar angle) contain the terms proportional to $\log r / r$ ( $r$ is the polar radius), 
which formally decay slower than the next linear term $\sim 1 / r$ as $r \longrightarrow+\infty$. This circumstance, in particular, gave reasons to Drela in work [38] and Giles and Drela in work [39] to include the nonlinear correction terms in their simplified far-field potential model for the compressible airfoil calculations. (Note, the entire series that represents the behavior at infinity of the potential function of a two-dimensional subsonic compressible flow has been accurately constructed by Ludford in work [40] using the hodograph plane techniques.)

Our two-dimensional DPM-based approach of [13], however, uses the full flow system; we never introduce the potential and we always consider only the linearized far-field flow. The accuracy and performance of the corresponding nonlocal ABC's are demonstrated by the numerical experiments, see our work $[14,15,18]$. These accuracy and performance are typically better than those of the standard methods. However, we should say that the investigated Mach numbers have never come really close to the transonic limit, we have always run our calculations in the range $M_{0} \lesssim 0.8$. Generally, retaining the second-order nonlinear terms in the compressible far-field model for twodimensional flows is most relevant to the case of Mach numbers close to one, $M_{0} \longrightarrow 1$, whereas the linear theory works best for $\delta \ll\left(1-M_{0}{ }^{2}\right)^{3 / 2}$ (see [37]), here $\delta$ can be regarded as, e.g., the airfoil thickness.

The situation with the compressible far-field expansion for three space dimensions is entirely different. Let us consider here the Kármán-Guderley equation (see [37])

$$
\frac{\partial^{2} \phi}{\partial x^{2}}+\frac{\partial^{2} \phi}{\partial \hat{y}^{2}}+\frac{\partial^{2} \phi}{\partial \hat{z}^{2}}=\frac{\gamma+1}{K} \frac{\partial \phi}{\partial x} \frac{\partial^{2} \phi}{\partial x^{2}}
$$

In equation (2.5), $\phi$ is the perturbation of the full potential $\Phi$ of the flow around a thin threedimensional wing so that

$$
\begin{gathered}
\frac{1}{u_{0}} \frac{\partial \Phi}{\partial x}=1+\delta^{2 / 3} \frac{\partial \phi}{\partial x}, \quad \frac{1}{u_{0}} \frac{\partial \Phi}{\partial y}=\delta \frac{\partial \phi}{\partial \tilde{y}}, \quad \frac{1}{u_{0}} \frac{\partial \Phi}{\partial z}=\delta \frac{\partial \phi}{\partial \tilde{z}}, \\
\tilde{y}=\delta^{1 / 3} y, \quad \tilde{y}=\delta^{1 / 3} y,
\end{gathered}
$$

$\delta$ is the wing thickness $\left(\delta \longrightarrow+0\right.$ along with $M_{0} \longrightarrow 1$ in the transonic limit $)$,

$$
K=\frac{1-M_{0}^{2}}{\delta^{2 / 3}}
$$

is the parameter of transonic similarity (the true linear theory corresponds to big values of $K$, see, e.g., [41]), the additional coordinate transformation is given by

$$
\hat{y}=\sqrt{\Pi} \tilde{y}, \quad \hat{z}=\sqrt{K} \tilde{z},
$$

$\gamma$ is still the ratio of specific heats, and the free stream is again aligned with the positive $x$ direction.

The far-field expansion for $\phi$ in the linear theory, i.e., when the right-hand side (RHS) of equation (2.5) is omitted, starts with the horseshoe vortex (see, e.g., [42])

$$
\phi_{1}=\frac{\hat{y}}{\hat{y}^{2}+\hat{z}^{2}}\left(1+\frac{x}{\hat{r}}\right)=\frac{(1+\cos \theta) \cos \varphi}{\hat{r} \sin \theta},
$$

where the spherical coordinates are introduced as 


$$
x=\hat{r} \cos \theta, \quad \hat{y}=\hat{r} \sin \theta \cos \varphi, \quad \hat{z}=\hat{r} \sin \theta \sin \varphi .
$$

Expression (2.7a) obviously has a singularity in the wake, i.e., along the line $\theta=0$. Clearly, the source term of order $1 / \hat{r}$ is not present in the far-field expansion because the surface of the wing is assumed closed. Therefore, the next term in the linear expansion should be proportional to $1 / \hat{r}^{2}$. We consider its general form $\phi_{2}=\sum_{l, m} \hat{r}^{-l-1} Y_{l}^{m}(\theta, \varphi), l=1, m=-1,0,1$, where the spherical functions $Y_{l}^{m}(\theta, \varphi)$ are given by $Y_{l}^{m}(\theta, \varphi)=\mathcal{P}_{l}^{m}(\cos \theta) \exp (i m \varphi)$, and $\mathcal{P}_{l}^{m}(\mu)=\left(1-\mu^{2}\right)^{m / 2} \mathcal{P}_{l}^{(m)}(\mu)$, $m \leq l$, here $\mathcal{P}_{l}(\mu)=\frac{1}{2^{l} l !} \frac{d^{l}}{d \mu^{l}}\left(\mu^{2}-1\right)^{l}$ are the Legendre polynomials. Using the real representation, we obtain the general form of the second term as

$$
\phi_{2}=a \frac{x}{\hat{r}^{3}}+b \frac{\hat{y}}{\hat{r}^{3}}+c \frac{\hat{z}}{\hat{r}^{3}}=a \frac{\cos \theta}{\hat{r}^{2}}+b \frac{\sin \theta \cos \varphi}{\hat{r}^{2}}+c \frac{\sin \theta \sin \varphi}{\hat{r}^{2}}
$$

where $a, b$, and $c$ are some arbitrary constants.

To obtain the nonlinear corrections due to the RHS of equation (2.5), one can substitute the linear terms (2.7) into this RHS and solve the resulting Poisson equation. For the purpose of simple demonstration we will do that separately for $\phi_{1}$ of $(2.7 \mathrm{a})$ and $\phi_{2}$ of $(2.7 \mathrm{~b})$ although the similar procedure can be carried out for any weighted sum of $\phi_{1}$ and $\phi_{2}$.

Substitution of (2.7a) into the RHS of equation (2.5) yields

$$
\frac{1}{\hat{r}^{2}} \frac{\partial}{\partial \hat{r}}\left(\hat{r}^{2} \frac{\partial \phi}{\partial \hat{r}}\right)+\frac{1}{\hat{r}^{2} \sin \theta} \frac{\partial}{\partial \theta}\left(\sin \theta \frac{\partial \phi}{\partial \theta}\right)+\frac{1}{\hat{r}^{2} \sin ^{2} \theta} \frac{\partial^{2} \phi}{\partial \varphi^{2}}=-\frac{\gamma+1}{K} \frac{3 \cos \theta \sin ^{2} \theta \cos ^{2} \varphi}{\hat{r}^{5}} .
$$

Note, the singularity of the potential (2.7a) in the wake (along $\theta=0$ ) vanishes with differentiation. The RHS of equation (2.8) can now be expanded with respect to the spherical functions; the corresponding finite Fourier series is, in fact, given by

$$
\cos \theta \sin ^{2} \theta \cos ^{2} \varphi=\frac{1}{5} Y_{1}^{0}(\theta, \varphi)-\frac{1}{5} Y_{3}^{0}(\theta, \varphi)+\frac{1}{30} Y_{3}^{2}(\theta, \varphi) .
$$

Taking into account that the spherical functions $Y_{l}^{m}$ are actually the eigenfunctions of the Beltrami operator on the sphere (two last terms on the left-hand side of equation (2.8)), we can separate the variables in equation (2.8) and reduce it to the finite family of one-dimensional equations with respect to the Fourier transformation $\hat{\phi}_{l, m} \equiv \hat{\phi}$ :

$$
\frac{d^{2} \hat{\phi}}{d \hat{r}^{2}}+\frac{2}{\hat{r}} \frac{d \hat{\phi}}{d \hat{r}}-\frac{l(l+1)}{\hat{r}^{2}} \hat{\phi}=\frac{A_{l}}{\hat{r}^{k+2}}, \quad l=1,3, \quad k=3
$$

The constants $A_{l}$ in (2.10) are, of course, inverse proportional to the transonic similarity parameter $K$ of $(2.6 \mathrm{~b})$, they also involve the coefficients of the expansion (2.9). The homogeneous counterpart to equation $(2.10)$ has two linearly independent solutions, $\hat{\phi}_{I}(\hat{r})=\hat{r}^{l}$ and $\hat{\phi}_{I I}(\hat{r})=\hat{r}^{-l-1}$. The solution $\hat{\phi}(\hat{r})$ to the nonhomogeneous equation (2.10) can therefore be found in the form

$$
\hat{\phi}(\hat{r})=c_{I}(\hat{r}) \hat{\phi}_{I}(\hat{r})+c_{I I}(\hat{r}) \hat{\phi}_{I I}(\hat{r}) \equiv c_{I}(\hat{r}) \hat{r}^{l}+c_{I I}(\hat{r}) \hat{r}^{-l-1}
$$

where the functions $c_{I}(\hat{r})$ and $c_{I I}(\hat{r})$ satisfy the system 


$$
\left[\begin{array}{cc}
\hat{\phi}_{I}(\hat{r}) & \hat{\phi}_{I I}(\hat{r}) \\
\frac{d \hat{\phi}_{I}(\hat{r})}{d \hat{r}} & \frac{d \hat{\phi}_{I I}(\hat{r})}{d \hat{r}}
\end{array}\right] \frac{d}{d \hat{r}}\left[\begin{array}{c}
c_{I}(\hat{r}) \\
c_{I I}(\hat{r})
\end{array}\right]=\left[\begin{array}{c}
0 \\
\frac{A_{l}}{\hat{r}^{k+2}}
\end{array}\right]
$$

Solving $(2.11 \mathrm{~b})$ with respect to $c_{I}(\hat{r})$ and $c_{I I}(\hat{r})$ yields

$$
c_{I}(\hat{r}) \sim \frac{1}{\hat{r}^{l+k}}, \quad c_{I I}(\hat{r}) \sim \frac{1}{\hat{r}^{-l+k-1}}
$$

provided that $l+k \neq 0$ and $-l+k \neq 1$, respectively. As both of the latter conditions are met for $l$ and $k$ from (2.10), we substitute the expressions (2.12) into equality (2.11a) and finally obtain the nonlinear correction due to the horseshoe potential of $(2.7 \mathrm{a})$ as

$$
\phi_{\mathbf{1}_{N L}} \sim \frac{1}{\hat{r}^{3}}
$$

The same type of derivation can be performed for the doublet potential of $(2.7 \mathrm{~b})$. Substituting $\phi_{2}$ of (2.7b) into the RHS of equation (2.5) one obtains the expression proportional to $\hat{r}^{-7}$ instead of $\hat{r}^{-5}$ in the RHS of equation (2.8). The expansion analogous to (2.9) will now contain $Y_{l}^{m}$ for $l=1,3,5$, and after the separation of variables the equation (2.10) will also change accordingly, we will have $k=5$ instead of $k=3$ and add $l=5$ to the set of wavenumbers. As a result, the nonlinear correction due to the potential $\phi_{2}$ of $(2.7 \mathrm{~b})$ can be shown to have the form

$$
\phi_{2_{N L}} \sim \frac{1}{\hat{r}^{5}}
$$

We see that the nonlinear correction $\phi_{1_{N L}}$ of (2.13a) decays at infinity two orders of magnitude faster than the term $\phi_{1}$ of (2.7a) that it originates from. Analogously, the nonlinear correction $\phi_{2_{N L}}$ of (2.13b) decays at infinity three orders of magnitude faster than the corresponding term $\phi_{2}$ of (2.7b). We therefore conclude, that unlike the two-dimensional case, the transonic nonlinear corrections are not required when analyzing the far field for three space dimensions. This conclusion basically coincides with the results of [37] saying that the far field around a thin three-dimensional finite-span wing is essentioally linear. In other words, we have shown that the small perturbations of the velocity potential of a three-dimensional compressible flow are described by the linear formulas in the far field even when $M_{0} \longrightarrow 1$. This justifies the far-field linearization of Section 2.1.1.

2.1.3. Outline of the Algorithm. Having introduced the linearized model (2.1), (2.2) for the far filed (see Section 2.1.1) we now obtain the combined problem: nonlinear inside the finite computational domain and linear on its infinite exterior. The nonlinear and linear parts of the problem are, of course, not independent. The interior and exterior solutions should match at the artificial boundary and consequently, the combined problem must be solved as a whole. Therefore, at the first glance the new problem is no easier than the original one from the standpoint of solving it numerically because it is still formulated on the unbounded domain. However, using the methodology of Calderon's projections and the DPM $[6,7,8]$, the exterior linear problem can be effectively reduced to a certain nonlocal relation formulated on the artificial boundary. The latter relation can serve as the desired $\mathrm{ABC}$ 's.

More precisely, we introduce a special space of (vector-)functions at the artificial boundary, it will be called the space of clear traces $[6,7,8]$. We also define a (pseudodifferential) projection operator that maps the space of clear traces onto itself. This operator will be analogous to 
Calderon's boundary projections. Under certain conditions, one can show that the element of the space of clear traces is actually a trace of some solution to the problem (2.1), (2.2) on the exterior domain if and only if this element belongs to the image of the aforementioned projection operator. The latter condition can, in fact, be written in the form of Calderon's boundary equation or the so-called boundary equation with projection (BEP) $[6,7,8]$. Its solutions will provide us with the complete boundary classification (in terms of the appropriate traces) of all those and only those $\boldsymbol{u}$ 's (see (2.1b) and (2.1c)) that solve (2.1), (2.2) outside the computational domain.

As we intend to set the ABC's for the discretized flow problem, the foregoing boundary classification of the exterior solutions will also be obtained in the discrete framework using the concept of finite-difference clear traces and finite-difference counterparts to Calderon's projections and generalized potentials $[6,7,8]$. Once we are able to calculate the image of Calderon's projection (i.e., the result of application of this operator to every given input), we can actually set the ABC's in a few different ways. Earlier (see $[13,14,15]$ ) we have been solving the corresponding BEP using some variational approach. Below, we follow the different path, namely, we implement the boundary projections directly as proposed first in [18]. In fact, applying the Calderon operator we update the missing boundary values on every cycle of the iteration procedure that is employed inside the computational domain.

2.2. Geometric Issues and the Basics of the Discrete Algorithm. The specific configuration of domains that we will be dealing with hereafter is shown in Figure 2.1.

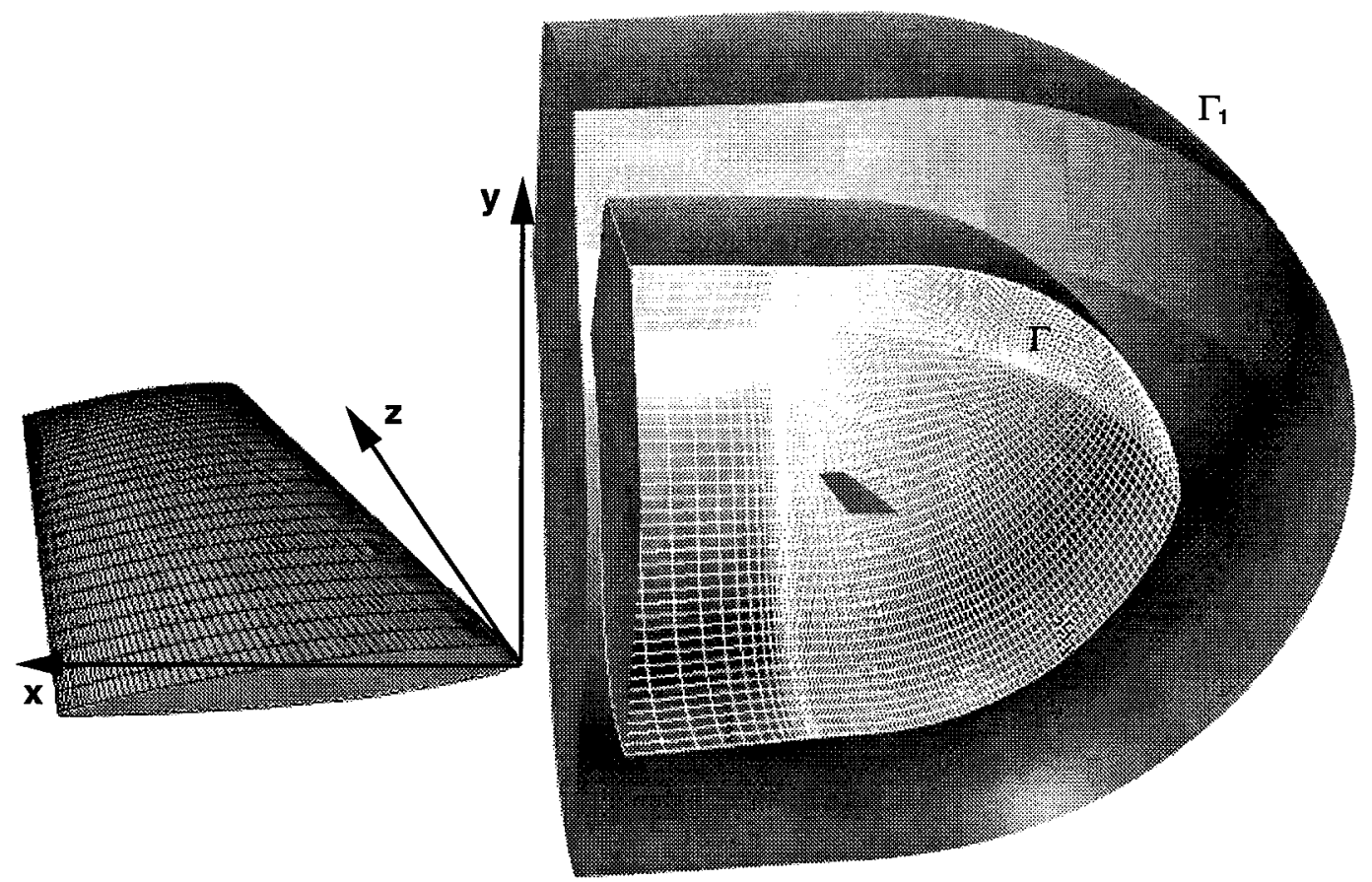

FIG. 2.1. Schematic geometric setup; the wing on the left is enlarged.

The actual structure displayed in this figure is the well-known test wing ONERA M6 (the wingtip is blunted, it is in the "hidden" area on the figure). The wing stretches span-wise along the Cartesian axis $z$ and is assumed symmetric with respect to the plane $z=0$. The uniform at infinity fluid flow is coming along the positive $x$ direction, which together with the symmetry of the wing implies the symmetry of the entire flow pattern with respect to $z=0$. 
The latter symmetry, in turn, means that the components $u^{i}$ of $\boldsymbol{u}, i=1, \ldots, 4$ for $(2.1 \mathrm{~b})$ and $i=1, \ldots, 5$ for $(2.1 \mathrm{c})$ (note, the $x$ projection of the velocity vector $u \equiv u^{i}$ for $i=2$ ) satisfy the following set of equalities for $i \neq 4$ :

$$
\begin{gathered}
\frac{\partial u^{i}}{\partial x}(\cdot,|z|)=\frac{\partial u^{i}}{\partial x}(\cdot,-|z|), \quad \frac{\partial u^{i}}{\partial y}(\cdot,|z|)=\frac{\partial u^{i}}{\partial y}(\cdot,-|z|), \quad \frac{\partial u^{i}}{\partial z}(\cdot,|z|)=-\frac{\partial u^{i}}{\partial z}(\cdot,-|z|), \\
\frac{\partial^{2} u^{i}}{\partial y^{2}}(\cdot,|z|)=\frac{\partial^{2} u^{i}}{\partial y^{2}}(\cdot,-|z|), \quad \frac{\partial^{2} u^{i}}{\partial z^{2}}(\cdot,|z|)=\frac{\partial^{2} u^{i}}{\partial z^{2}}(\cdot,-|z|), \quad \frac{\partial^{2} u^{i}}{\partial y \partial z}(\cdot,|z|)=-\frac{\partial^{2} u^{i}}{\partial y \partial z}(\cdot,-|z|),
\end{gathered}
$$

which, in particular, implies

$$
\frac{\partial u^{i}}{\partial z}(\cdot, 0)=0, \quad \frac{\partial^{2} u^{i}}{\partial y \partial z}(\cdot, 0)=0, \quad \text { for } i \neq 4
$$

and the following set of equalities for $i=4$ :

$$
\begin{gathered}
\frac{\partial u^{i}}{\partial x}(\cdot,|z|)=-\frac{\partial u^{i}}{\partial x}(\cdot,-|z|), \quad \frac{\partial u^{i}}{\partial y}(\cdot,|z|)=-\frac{\partial u^{i}}{\partial y}(\cdot,-|z|), \quad \frac{\partial u^{i}}{\partial z}(\cdot,|z|)=\frac{\partial u^{i}}{\partial z}(\cdot,-|z|), \\
\frac{\partial^{2} u^{i}}{\partial y^{2}}(\cdot,|z|)=-\frac{\partial^{2} u^{i}}{\partial y^{2}}(\cdot,-|z|), \quad \frac{\partial^{2} u^{i}}{\partial z^{2}}(\cdot,|z|)=-\frac{\partial^{2} u^{i}}{\partial z^{2}}(\cdot,-|z|), \quad \frac{\partial^{2} u^{i}}{\partial y \partial z}(\cdot,|z|)=\frac{\partial^{2} u^{i}}{\partial y \partial z}(\cdot,-|z|),
\end{gathered}
$$

which yields

$$
\begin{aligned}
u^{i}(\cdot, 0)=0, \quad \frac{\partial u^{i}}{\partial x}(\cdot, 0) & =0, \quad \frac{\partial u^{i}}{\partial y}(\cdot, 0)=0, \\
\frac{\partial^{2} u^{i}}{\partial y^{2}}(\cdot, 0) & =0, \quad \frac{\partial^{2} u^{i}}{\partial z^{2}}(\cdot, 0)=0, \quad \text { for } i=4 .
\end{aligned}
$$

Relations (2.14), (2.15) will be used in Section 2.4 when constructing the discretization for the linearized system.

The flow equations are integrated numerically on a curvilinear grid generated around the wing. The grid shown in Figure 2.1 is a one-block $\mathrm{C}$-O type grid; it this paper we will use the grids of this type only. The surface designated $\Gamma$ on Figure 2.1 is actually the external set of nodes of the C-O grid, i.e., the artificial boundary. Henceforth, we will also need the notation $D_{\text {in }}$ for the interior of T, i.e., for the finite computational domain, and the notation $D_{e x}$ for the infinite exterior of $\Gamma$.

The curve $\Gamma_{1} \subset D_{e x}$ (see Figure 2.1) actually represents the set of ghost nodes (or centers of the ghost cells for the case of finite-volume discretization), it can also be thought of as the outermost set of nodes of the original $\mathrm{C}-\mathrm{O}$ grid; the surface $\Gamma$ then becomes the penultimate set of nodes. We will further assume that the linearization $(2.1)$ is valid in $D_{e x}$, i.e., outside $\Gamma$, so that $\Gamma_{1}$ belongs already to the linear zone. The actual admissible size of $D_{i n}$ such that the perturbations can be considered sufficiently small and therefore the assumption of the linearity in $D_{\epsilon x}$ would hold, is, of course, unknown ahead of time. We verify the validity of linearization in $D_{e x}$ a posteriori, through the series of numerical experiments, see Section 3. 
Clearly, when the stencil of the scheme used inside $D_{\text {in }}$ is applied to any node from $\Gamma$, it generally requires some ghost cell data. Note, for the second-order central difference schemes (like those employed in the code FLOMG, see [10,11, 12] and TLNS3D, see [21]) the consideration of only one layer of ghost cells $\Gamma_{1}$ is sufficient, but the case when the stencil is more extensive and consequently, more ghost cells are required, can be treated similarly. Unless the missing ghost cell data are provided, i.e., obtained with the help of the $A B C$ 's, the discrete system solved inside the computational domain appears subdefinite, in other words, it has less equations than it has unknowns. As mentioned in Section 2.1.3, the apparatus of the DPM $[6,7,8]$ gives us the complete boundary characterization of the traces of exterior linear solutions. Since the linear zone $D_{e x}$ stretches from $\Gamma$ to infinity and contains $\Gamma_{1}$, the following approach appears most natural for setting the ABC's. First, the data provided from inside $D_{i n}$ are subjected to the projection operation. The resulting projection will by definition admit a complement on $D_{e x}$ that solves (2.1), (2.2). The latter complement can be calculated in the form of a generalized potential and considered on $\Gamma_{1}$. Altogether, this procedure yields the missing relations between the values of the solution on $\Gamma$ and $\Gamma_{1}$. In other words, it provides for a desired closure to the discrete system solved inside $D_{i n}$, or the ABC's. Typically, the solution algorithm inside $D_{i n}$ involves some pseudo-time iterations (see Section 3); then, the foregoing closing procedure is applied on every iteration cycle, more precisely, every time the ghost cells need to be updated in order to advance the next time step.

We now proceed to the description of the generalized potentials and boundary projections, as well as their finite-difference counterparts, that are required for setting the DPM-based ABC's. Note, if the potentials and projections are calculated for exactly the operator $\boldsymbol{L}$ of (2.1a) that operates on the functions $\boldsymbol{u}$ defined on the entire inffinite domain $D_{e x}$ and satisfying boundary conditions (2.2), then the corresponding BEP appears equivalent to the original linear problem $(2.1),(2.2)$ (see $[6,7,8]$ ). We, however, will have to introduce some simplifications and to carry out the DPM-based procedure for a certain approximation of problem (2.1), (2.2) (see Sections 2.3 and 2.4) rather than for this problem itself. Nonetheless, the corresponding approximate solution can be made as close to the original one as initially prescribed (see [18] and below for more detail). Therefore, within the accuracy of far-field linearization the resulting ABC's can be made as close to the exact ones as desired.

2.3. Foundations of the DPM-based ABC's. Here, we will first formulate and solve the so-called auxiliary problem (AP) for the inhomogeneous version of system (2.1) with boundary conditions (2.2). This AP will be the central element of our construction of Calderon's generalized potentials and boundary projections. In fact, the solution of the AP can be thought of as a substitute for the convolution with the fundamental solution in classical potential theory.

2.3.1. Infinite-Domain AP. Let us consider a compactly supported vector-function $\left\{f^{i}\right\} \equiv$ $\boldsymbol{f} \equiv \boldsymbol{f}(x, y, z)$, supp $\boldsymbol{f} \subseteq D_{\text {in }}$, of dimension four $(i=1, \ldots, 4$ for $(2.1 \mathrm{~b}))$ or five $(i=1, \ldots, 5$ for (2.1c)); in the meantime, we do not specify the concrete form of $f$. This function $f$ will be the right-hand side for the AP. The AP is initially formulated on the entire space $R^{3}$; namely, we will be looking for a solution $\boldsymbol{u}$ of system

$$
L u=f
$$

that meets boundary condition $(2.2)$ at infinity.

Note, when discussing regular solutions below we assume, if necessary, that the functions involved can be represented in the form of their Fourier integrals.

Proposition 2.1. Let $\boldsymbol{f}$ be a compactly supported distribution, $\boldsymbol{f} \in \mathcal{D}^{\prime}\left(R^{3}\right)$, supp $\boldsymbol{f} \subset D_{\text {in }}$. Then, system (2.16), (2.16) is solvable in the Schwartz space $\mathcal{D}^{\prime}\left(R^{3}\right)$. 
To justify proposition 2.1 , we use the standard methodology based on application of the Fourier transform over the entire $R^{3}$ (see, e.g., [43]). Clearly, to obtain the solvability of $(2.16)$ in $\mathcal{D}^{\prime}\left(R^{3}\right)$ it is sufficient to make sure that the inverse symbol of the operator $L$ (see (2.1)) belongs locally to $L_{1}\left(R^{3}\right)$. Denoting the dual (Fourier) variables to $(x, y, z)$ by $(\xi, \eta, \zeta)$, we can write the symbol $\boldsymbol{Q}$ of $L$ as

$$
\boldsymbol{Q}=i \xi \boldsymbol{C}+i \eta \boldsymbol{D}+i \zeta \boldsymbol{E}-\eta^{2} \boldsymbol{F}-\zeta^{2} \boldsymbol{H}-\eta \zeta \boldsymbol{J}
$$

Then, the entrees $\tilde{q}_{j k}=\tilde{q}_{k j}, j=1, \ldots, 4, k=1, \ldots, 4$, of the inverse symbol $\boldsymbol{Q}^{-1}$ for the incompressible case $(2.1 \mathrm{~b})$ are given by

$$
\begin{array}{rlrl}
\tilde{q}_{11}=\frac{i \xi+\frac{\eta^{2}+\zeta^{2}}{R e}}{\varrho^{2}}, & \tilde{q}_{12}=\frac{-i \xi}{\varrho^{2}}, \quad \tilde{q}_{13}=\frac{-i \eta}{\varrho^{2}}, & \tilde{q}_{14}=\frac{-i \zeta}{\varrho^{2}} \\
\tilde{q}_{22}=\frac{\eta^{2}+\zeta^{2}}{\left(i \xi+\frac{\eta^{2}+\zeta^{2}}{R e}\right) \varrho^{2}}, & \tilde{q}_{23}=\frac{-\xi \eta}{\left(i \xi+\frac{\eta^{2}+\zeta^{2}}{R e}\right) \varrho^{2}}, & \tilde{q}_{24}=\frac{-\xi \zeta}{\left(i \xi+\frac{\eta^{2}+\zeta^{2}}{R e}\right) \varrho^{2}}, \\
\tilde{q}_{33}=\frac{\xi^{2}+\zeta^{2}}{\left(i \xi+\frac{\eta^{2}+\zeta^{2}}{R e}\right) \varrho^{2}}, & \tilde{q}_{34}=\frac{-\eta \zeta}{\left(i \xi+\frac{\eta^{2}+\zeta^{2}}{R e}\right) \varrho^{2}}, \\
\tilde{q}_{44} & =\frac{\xi^{2}+\eta^{2}}{\left(i \xi+\frac{\eta^{2}+\zeta^{2}}{R e}\right) \varrho^{2}},
\end{array}
$$

where $\varrho^{2}=\xi^{2}+\eta^{2}+\zeta^{2}$. From (2.18) one can see that $\boldsymbol{Q}^{-1}$ has only one real singularity, it is located in the origin, $(\xi, \eta, \zeta)=(0,0,0)$. Clearly, all $\tilde{q}_{1 k}, k=1, \ldots, 4$, are absolutely integrable near the origin and consequently, $\tilde{q}_{1 k} \in L_{1}^{\text {loc }}\left(R^{3}\right)$ for $k=1, \ldots, 4$. As for the other $\tilde{q}_{j k}$, we introf . o the polar coordinates: $\xi=\varrho \cos \theta, \eta=\varrho \sin \theta \cos \varphi, \zeta=\varrho \sin \theta \sin \varphi, 0 \leq \theta \leq \pi, 0 \leq \varphi<2 \pi$. . d notice that for sufficiently small $\varrho$ 's

$$
\frac{\varrho^{2}}{R e^{2}} \leq \cos ^{2} \theta+\frac{\varrho^{2}}{R e^{2}} \sin ^{4} \theta, \quad 0 \leq \theta \leq \pi
$$

For $j=2, \ldots, 4$ and $k=j, \ldots, 4$, estimate (2.19) immediately yields

$$
\left|\tilde{q}_{j k}\right| \leq \operatorname{const} \frac{\varrho^{2}}{\left|i \xi+\frac{\eta^{2}+\zeta^{2}}{R e}\right| \varrho^{2}}=\frac{\text { const }}{\varrho\left(\cos ^{2} \theta+\frac{\varrho^{2}}{R e^{2}} \sin ^{4} \theta\right)^{1 / 2}} \leq \operatorname{const} \frac{R e}{\varrho^{2}},
$$

and we therefore conclude that $\tilde{q}_{j k} \in L_{1}^{\text {loc }}\left(R^{3}\right)$ for all $j=1, \ldots, 4, k=1, \ldots, 4$. Thus, we have shown that proposition 2.1 does hold for the incompressible case (see (2.1b)). Note, similar proof for the two-dimensional compressible case can be found in [17].

Proposition 2.2. Let $f \in L_{1}\left(R^{3}\right)$. Then, equation (2.16), (2.1b) may have no more than one regular solution $\boldsymbol{u} \in \mathcal{D}^{\prime}\left(R^{3}\right)$ that vanishes at infinity, i.e. satisfies (2.2).

Indeed, the Fourier transformation $\hat{f}$ of the RHS is continuous on $R^{3}$ in this case. The regular solution $\boldsymbol{u}$ to $(2.16),(2.2)$ is given by the inverse Fourier transform: $\boldsymbol{u}=\left(\boldsymbol{Q}^{-1} \hat{\boldsymbol{f}}\right)^{\curvearrowright}$. Since $\boldsymbol{Q}^{-1} \hat{f}$ has only one real singularity (in the origin), then any other solution can differ from $\boldsymbol{u}$ by no more than an inverse Fourier transformation of a distribution concentrated in the origin. The latter can be nothing but a sum of $\delta$-functions and their derivatives, which correspond to polynomials after the Fourier transform. Therefore, proposition 2.2, which is actually a statement of conditional uniqueness, has been justified. Note that we have been able to establish uniqueness so easily because 
the inverse symbol $\boldsymbol{Q}^{-1}$ has only one isolated real singular point. This, for example, would not be the case for the Euler equations, which can be obtained by formally letting $R e^{-1}=0$.

Proposition 2.3. Let $f$ be compactly supported and $f \in L_{2}\left(R^{3}\right)$. Then, equation (2.16), (2.1b) has a solution $\boldsymbol{u}=\boldsymbol{u}^{I}+\boldsymbol{u}^{I I}$, where $\boldsymbol{u}^{I}$ is infinitely smooth on $R^{3}$, i.e., $\boldsymbol{u}^{I} \in C^{\infty}\left(R^{3}\right)$, and satisfies boundary condition (2.2), and $\boldsymbol{u}^{I I} \in L_{2}\left(R^{3}\right)$. Moreover, for any $\epsilon>0$, one can always choose the representation $\boldsymbol{u}=\boldsymbol{u}^{I}+\boldsymbol{u}^{I I}$ so that $\left\|\boldsymbol{u}^{I I}\right\|_{L_{2}\left(R^{3}\right)}<\epsilon$.

Consider a partition of unity $1=g_{0}+g_{\overline{0}}$, where both (scalar) functions $g_{0}$ and $g_{\overline{0}}$ are infinitely smooth on $R^{3}, g_{0} \equiv 1$ on a ball $U_{R_{0}}$ centered in the origin with the fixed radius $R_{0}$, and $g_{0} \equiv 0$ outside a bigger ball $U_{R_{0}+\mu}$ with the radius $R_{0}+\mu, \mu>0$. Clearly, as $f \in L_{2}\left(R^{3}\right)$ and $f$ is compactly supported, then $f \in L_{1}\left(R^{3}\right)$. The solution $\boldsymbol{u}$ is given by the inverse Fourier transform: $\boldsymbol{u}=\left(\boldsymbol{Q}^{-1} \hat{\boldsymbol{f}}\right)^{\curvearrowright}=\left(\boldsymbol{Q}^{-1} g_{0} \hat{\boldsymbol{f}}\right)^{\curvearrowright}+\left(\boldsymbol{Q}^{-1} g_{\overline{0}} \hat{\boldsymbol{f}}\right)^{\curlyvee}$. The first term of the foregoing sum, $\boldsymbol{u}^{I} \equiv\left(\boldsymbol{Q}^{-1} g_{0} \hat{f}\right)^{\curvearrowright}$, is obviously an inverse Fourier transformation of a function from $L_{1}\left(R^{3}\right)$. Moreover, for any polynomial $P \equiv P(\xi, \eta, \zeta): P Q^{-1} g_{0} \hat{\boldsymbol{f}} \in L_{1}\left(R^{3}\right)$. Therefore, $\boldsymbol{u}^{I} \in C^{\infty}\left(R^{3}\right)$ and $\boldsymbol{u}^{I}$ meets boundary condition (2.2). The second term, $\boldsymbol{u}^{I I} \equiv\left(\boldsymbol{Q}^{-1} g_{\overline{0}} \hat{f}\right)^{2}$, is an inverse Fourier transformation of a function from $L_{2}\left(R^{3}\right)$ because $\hat{f} \in L_{2}\left(R^{3}\right)$ and $\boldsymbol{Q}^{-1}$ is bounded when $\varrho \longrightarrow+\infty$ as can be clearly seen from expressions (2.18). (The Fourier transform in this case can be regarded in the sense of Plancherel.) Therefore, $\boldsymbol{u}^{I I} \in L_{2}\left(R^{3}\right)$. Clearly, both $\boldsymbol{u}^{I}$ and $\boldsymbol{u}^{I I}$ depend on the choice of the partition of unity $1=g_{0}+g_{\overline{0}}$, i.e., on the choice of $R_{0}$. Since $\hat{\boldsymbol{u}}^{I I} \equiv \boldsymbol{Q}^{-1} g_{\overline{0}} \hat{f} \in L_{2}\left(R^{3}\right)$ for any $R_{0}$, then $\left\|Q^{-1} g_{\overline{0}} \hat{f}\right\|_{L_{2}\left(R^{3}\right)} \longrightarrow 0$ as $R_{0} \longrightarrow+\infty$ and consequently, $\left\|\boldsymbol{u}^{I I}\right\|_{L_{2}\left(R^{2}\right)} \longrightarrow 0$ as $R_{0} \longrightarrow+\infty$. This concludes the proof of proposition 2.3.

Proposition 2.4. Let $\hat{f}$ be the Fourier transformation of $f$ on $R^{3}$ and $\hat{f} \in L_{1}\left(R^{3}\right)$. Then, equation (2.16), (2.16) has a continuous solution $u$ on $R^{3}$ that meets boundary condition (2.2).

The statement of proposition 2.4 is obvious as in this case $Q^{-1} \hat{f} \in L_{1}\left(R^{3}\right)$.

In our further constructions, however, we will not always be able to guarantee the inclusion $\hat{f} \in L_{\mathbf{1}}\left(R^{3}\right)$ as required in proposition 2.4. Typically, supp $f \subseteq D_{i n}$ and we may also assume that $f$ is sufficiently smooth on $D_{i n}$. On the other hand, we do not generally require the differentiability of $f$ on the entire $R^{3} ; f$ and its derivatives may have the discontinuities (of the first kind) on the surface $\Gamma \equiv \partial D_{i n}$. For any RHS $\boldsymbol{f}$ of this type, we will make sure that when we successively approximate $f$ by the smooth functions $\boldsymbol{f}_{n}$ the corresponding smooth solutions $\boldsymbol{u}_{n}$ in a certain sense converge to the solution $\boldsymbol{u}$ guaranteed by proposition 2.3 .

Consider a sequence $f_{n}, n=1,2, \ldots$, of infinitely smooth compactly supported on $D_{\text {in }}$ functions that converges to $\boldsymbol{f}$ in the sense of $L_{2}\left(D_{i n}\right):\left\|f-\boldsymbol{f}_{n}\right\|_{L_{2}\left(D_{i n}\right)} \equiv\left\|f-\boldsymbol{f}_{n}\right\|_{L_{2}\left(R^{3}\right)} \longrightarrow 0$ as $n \longrightarrow+\infty$. (The sequence $f_{n}$ always exists because $f \in L_{2}\left(D_{i n}\right)$ and the space $\mathcal{D}\left(D_{i n}\right)$ of all compactly supported infinitely smooth functions on $D_{i n}$ is everywhere dense in $L_{2}\left(D_{i n}\right)$, see, e.g., [43].) The Fourier transformation $\hat{\boldsymbol{f}}_{n}$ of any $\boldsymbol{f}_{n} \in \mathcal{D}\left(D_{i n}\right)$ is infinitely smooth on $R^{3}$ and decays at infinity faster than any power of $r^{-1}$ with all its derivatives. (Fourier transform in the sense of Plancherel obviously coincides here with the standard transform in the sense of $L_{1}$.) Therefore, for any polynomial $P \equiv P(\xi, \eta, \zeta): P Q^{-1} \hat{\boldsymbol{f}}_{n} \in L_{1}\left(R^{3}\right)$ and consequently, the solution $\boldsymbol{u}_{n}$ to the system $\boldsymbol{L} \boldsymbol{u}_{n}=\boldsymbol{f}_{n}$ is also infinitely smooth on $R^{3}, \boldsymbol{u}_{n} \in C^{\infty}\left(R^{3}\right)$, and satisfies boundary condition (2.2). We now consider the same partition of unity $1=g_{0}+g_{\overline{0}}$ as used when proving proposition $2.3 \cdot \boldsymbol{u}_{n}=\left(\boldsymbol{Q}^{-1} \hat{\boldsymbol{f}}_{n}\right)^{\vee}=$ $\boldsymbol{u}_{n}^{I}+\boldsymbol{u}_{n}^{I I}$, where $\boldsymbol{u}_{n}^{I}=\left(\boldsymbol{Q}^{-1} g_{0} \hat{\boldsymbol{f}}_{n}\right)^{\curlyvee}$ and $\boldsymbol{u}_{n}^{I I}=\left(\boldsymbol{Q}^{-1} g_{\overline{0}} \hat{f}_{n}\right)^{\curlyvee} ;$ clearly, both $\boldsymbol{u}_{n}^{I}, \boldsymbol{u}_{n}^{I I} \in C^{\infty}\left(R^{3}\right)$ and both $\boldsymbol{u}_{n}^{I}, \boldsymbol{u}_{n}^{I I} \longrightarrow 0$ when $r \longrightarrow \infty$. As $\boldsymbol{f}_{n} \stackrel{L_{2}\left(R^{3}\right)}{\longrightarrow} \boldsymbol{f}$, then $\hat{\boldsymbol{f}}_{n} \stackrel{L_{2}\left(R^{3}\right)}{\longrightarrow} \hat{\boldsymbol{f}}$ and $g_{0} \hat{\boldsymbol{f}}_{n} \stackrel{L_{2}\left(U_{R_{0}}\right)}{\longrightarrow}$ $g_{0} \hat{f}$. Consequently, $g_{0} \hat{f}_{n} \stackrel{L_{1}\left(U_{R_{0}}\right)}{\longrightarrow} g_{0} \hat{f}$ and therefore $g_{0} \hat{f}_{n} \stackrel{L_{1}\left(R^{3}\right)}{\longrightarrow} g_{0} \hat{f}$. Since $Q^{-1} \in L_{1}^{l o c}\left(R^{3}\right)$, then $\boldsymbol{Q}^{-1} g_{0} \hat{\boldsymbol{f}}_{n} \stackrel{L_{1}\left(R^{3}\right)}{\longrightarrow} \boldsymbol{Q}^{-1} g_{0} \hat{\boldsymbol{f}}$ and also for any polynomial $P \equiv P(\xi, \eta, \zeta): P Q^{-1} g_{0} \hat{\boldsymbol{f}}_{n} \stackrel{L_{1}\left(R^{3}\right)}{\longrightarrow}$ $P Q^{-1} g_{0} \hat{f}$. Therefore, $\boldsymbol{u}_{n}^{I}$ uniformly converges to $\boldsymbol{u}^{I}=\left(\boldsymbol{Q}^{-1} g_{0} \hat{f}\right)^{\curlyvee}$ on $R^{3}$ with all its derivatives, 
$\frac{\partial^{\alpha+\beta+\gamma}}{\partial x^{\alpha} \partial y^{\beta \partial z^{\gamma}}} \boldsymbol{u}_{n}^{I} \Longrightarrow \frac{\partial^{\alpha+\beta+\gamma}}{\partial x^{\alpha} \partial y^{\beta} \partial z^{\gamma}} \boldsymbol{u}^{I}$ as $n \longrightarrow+\infty$ (see, e.g., [44]). As for the second term, obviously $g_{0} \hat{\boldsymbol{f}}_{n} \stackrel{L_{2}\left(R^{3}\right)}{\longrightarrow} g_{\overline{0}} \hat{\boldsymbol{f}}$ and consequently, $\boldsymbol{Q}^{-1} g_{\overline{0}} \hat{\boldsymbol{f}}_{n} \stackrel{L_{2}\left(R^{3}\right)}{\longrightarrow} \boldsymbol{Q}^{-1} g_{\overline{0}} \hat{\boldsymbol{f}}$. Therefore, $\boldsymbol{u}_{n}^{I I} \stackrel{L_{2}\left(R^{3}\right)}{\longrightarrow} \boldsymbol{u}^{I I}$ as $n \longrightarrow+\infty$. Thus, we have justified

Proposition 2.5. Let $\operatorname{supp} f \subseteq D_{\text {in }}$ and $f \in L_{2}\left(D_{\text {in }}\right)$. For any $f_{n} \in \mathcal{D}\left(D_{\text {in }}\right)$, the solution $\boldsymbol{u}_{n}$ to the system $\boldsymbol{L} \boldsymbol{u}_{n}=\boldsymbol{f}_{n}$ (see (2.1b)) satisfies boundary conditions (2.2) and $\boldsymbol{u}_{n} \in C^{\infty}\left(R^{3}\right)$. Moreover, if the sequence $\boldsymbol{f}_{n} \in \mathcal{D}\left(D_{i n}\right), n=1,2, \ldots$, converges to $f$ in the sense of $L_{2}, \boldsymbol{f}_{n} \stackrel{L_{2}\left(R^{3}\right)}{\longrightarrow} \boldsymbol{f}$, then each solution $\boldsymbol{u}_{n}$ can be represented as a sum of two terms, $\boldsymbol{u}_{n}=\boldsymbol{u}_{n}^{I}+\boldsymbol{u}_{n}^{I I}$, where $\boldsymbol{u}_{n}^{I}, \boldsymbol{u}_{n}^{I I} \in$ $C^{\infty}\left(R^{3}\right), \boldsymbol{u}_{n}^{I}, \boldsymbol{u}_{n}^{I I} \longrightarrow 0$ as $r \longrightarrow \infty, \frac{\partial^{\alpha+\beta+\gamma}}{\partial x^{\alpha} \partial y^{\beta} \partial z^{\gamma}} \boldsymbol{u}_{n}^{I} \Longrightarrow \frac{\partial^{\alpha+\beta+\gamma}}{\partial x^{\alpha} \partial y^{\beta} \partial z^{\gamma}} \boldsymbol{u}^{I}$ and $\boldsymbol{u}_{n}^{I I} \stackrel{L_{2}\left(R^{3}\right)}{\longrightarrow} \boldsymbol{u}^{I I}$ as $n \longrightarrow$ $+\infty$. Here $\boldsymbol{u}^{I}$ and $\boldsymbol{u}^{I I}$ are the same as in proposition 2.3.

2.3.2. Finite-Domain AP. To implement the DPM-based ABC's in practice (Section 3), we will need to be able to actually calculate the solution to the auxiliary problem. Since the formulation of the AP from Section 2.3.1 still involves infinite domain, we replace it by the approximate finitedomain formulation that allows easy numerical solution.

As any linear system of PDE's with constant coefficients, (2.16) admits the separation of variables in the Cartesian coordinates. Therefore, we implement the Fourier transform in the cross-stream and span-wise directions:

$$
\begin{aligned}
& \hat{\boldsymbol{u}}(x, \eta, \zeta)=\frac{1}{2 \pi} \iint_{-\infty}^{\infty} \boldsymbol{u}(x, y, z) e^{-i \eta y-i \zeta z} d y d z \\
& \hat{\boldsymbol{f}}(x, \eta, \zeta)=\frac{1}{2 \pi} \iint_{-\infty}^{\infty} \boldsymbol{f}(x, y, z) e^{-i \eta y-i \zeta z} d y d z
\end{aligned}
$$

and obtain a family of one-dimensional systems

$$
C \frac{d \hat{\boldsymbol{u}}}{d x}+\left(i \eta \boldsymbol{D}+i \zeta \boldsymbol{E}-\eta^{2} \boldsymbol{F}-\zeta^{2} \boldsymbol{H}-\eta \zeta \boldsymbol{J}\right) \hat{\boldsymbol{u}}=\hat{\boldsymbol{f}}
$$

that we consider on the entire line $-\infty<x<\infty$ for all $(\eta, \zeta) \in R^{2}$. Each system (2.21) is supplemented by the boundary condition

$$
|\hat{\boldsymbol{u}}(x, \eta, \zeta)| \leq \text { const for } \quad-\infty<x<\infty,
$$

which actually implies $|\hat{\boldsymbol{u}}(x, \eta, \zeta)| \longrightarrow 0$ as $|x| \longrightarrow \infty$ (compare to (2.2)) if there are no zeros among the eigenvalues of the matrix $\boldsymbol{Q} \equiv \boldsymbol{C}^{-1}\left(i \eta \boldsymbol{D}+i \zeta \boldsymbol{E}-\eta^{2} \boldsymbol{F}-\zeta^{2} \boldsymbol{H}-\eta \zeta \boldsymbol{J}\right)$. The only special case, for which the decay of $\hat{\boldsymbol{u}}(x, \eta, \zeta)$ when $|x| \longrightarrow \infty$ cannot be guaranteed, is $(\eta, \zeta)=(0,0)$; therefore, we generally set the boundary conditions in the form (2.22). It, however, has been shown in [13] that after the inverse Fourier transform the solution $\boldsymbol{u}$ will still vanish as $|x|$ increases.

Note that although designated by the same notations, $\hat{\boldsymbol{u}}$ and $\hat{f}$ in formulas $(2.20)$, as well as $\boldsymbol{Q}$, are not the same here as in the previous section. Indeed, the direction $x$ has been left out when calculating the Fourier transformations (2.20). This has been done because the natural spatial anisotropy prescribed by the direction of the free stream exists in our model and therefore the stream-wise coordinate $x$ will be given a special treatment in the finite-domain AP. 
Generally, the solution $\hat{u}(x, \eta, \zeta)$ to problem $(2.21),(2.22)$ can be found as a convolution

$$
\hat{\boldsymbol{u}}(x, \eta, \zeta)=\int_{-\infty}^{\infty} \boldsymbol{G}_{1}\left(x-x^{\prime}, \eta, \zeta\right) \hat{\boldsymbol{f}}\left(x^{\prime}, \eta, \zeta\right) d x^{\prime}
$$

with the corresponding one-dimensional fundamental solution $\boldsymbol{G}_{1}(x, \eta, \zeta)$. Then, the solution $\boldsymbol{u}$ to $\boldsymbol{L} \boldsymbol{u}=\boldsymbol{f}$ can be restored by means of the inverse Fourier transform, which eventually yields:

$$
\begin{aligned}
\boldsymbol{u}(x, y, z)= & \frac{1}{(2 \pi)^{2}} \int_{-\infty}^{\infty} \int_{-\infty} e^{i y \eta+i z \zeta} \int_{-\infty}^{\infty} \boldsymbol{G}_{1}\left(x-x^{\prime}, \eta, \zeta\right) \\
& \iint_{-\infty}^{\infty} \boldsymbol{f}\left(x, y^{\prime}, z^{\prime}\right) e^{-i \eta y^{\prime}-i \zeta z^{\prime}} d y^{\prime} d z^{\prime} d x^{\prime} d \eta d \zeta .
\end{aligned}
$$

Now, let us consider the new formulation of the AP that is periodic in both cross-stream and span-wise directions. Specifically, we introduce the periods $Y$ and $Z$ for the coordinates $y$ and $z$, respectively, replace the Fourier integrals by the Fourier series, and instead of (2.24) obtain

$$
\begin{aligned}
\boldsymbol{u}_{Y Z}(x, y, z)= & \frac{1}{Y Z} \sum_{k_{z}=-\infty}^{k_{z}=\infty} \sum_{k_{y}=-\infty}^{k_{y}=\infty} e^{i \frac{2 \pi k_{y}}{Y}+i z \frac{2 \pi k_{z}}{Z}} \int_{-\infty}^{\infty} \boldsymbol{G}_{1}\left(x-x^{\prime}, \frac{2 \pi k_{y}}{Y}, \frac{2 \pi k_{z}}{Z}\right) \\
& \iint_{-\infty}^{\infty} \boldsymbol{f}\left(x, y^{\prime}, z^{\prime}\right) e^{-i \frac{2 \pi k_{y}}{Y} y^{\prime}-i \frac{2 \pi k_{z}}{Z} z^{\prime}} d y^{\prime} d z^{\prime} d x^{\prime} .
\end{aligned}
$$

In our previous work ( two-dimensional case. It has been shown that for any fixed-size subdomain the periodic solution will converge to the original nonperiodic solution as the period increases. These results can be transferred to the case of three space dimensions without changes. Namely, let $Y_{0}$ and $Z_{0}$ be fixed. Then,

$$
\begin{gathered}
\boldsymbol{u}_{Y Z}(x, y, z) \longrightarrow \boldsymbol{u}(x, y, z) \text { as } \quad(Y, Z) \longrightarrow(+\infty,+\infty), \\
\text { when } \quad-\frac{Y_{0}}{2}<y<\frac{Y_{0}}{2}, \quad-\frac{Z_{0}}{2}<z<\frac{Z_{0}}{2} .
\end{gathered}
$$

The convergence considered in (2.26) is typically uniform. The convergence for the derivatives can also be established under some additional conditions (see [18]). Finally, as we are going to solve the AP by a finite-difference method (Section 3 ), certain relations between the period(s) and the grid size(s) should hold, see $[13,17]$ for more detail. We also note that the convergence on a fixed-size domain is sufficient for our purposes because for constructing the ABC's we will need to know the solution of the AP only on some neighborhood of the artificial boundary.

Thus, we have replaced the original infinite-domain AP by the new problem formulated on the beam-shaped domain $[-\infty, \infty] \times[-Y / 2, Y / 2] \times[-Z / 2, Z / 2]$. This domain is still infinite in the stream-wise direction. To make the entire formulation truly finite, we first introduce some interval $[0, X]$ so that $[0, X] \times[-Y / 2, Y / 2] \times[-Z / 2, Z / 2] \supset \Gamma_{1}$. Consequently, systems $(2.21)$ will be homogeneous outside $[0, X]$ for all $\left(\eta_{k_{y}}, \zeta_{k_{z}}\right) \equiv\left(\frac{2 \pi k_{y}}{Y}, \frac{2 \pi k_{z}}{Z}\right)$. Then, boundary condition 


$$
\left[\prod_{\Re \lambda(k)<0}(\boldsymbol{Q}(\boldsymbol{k})-\lambda(k) \boldsymbol{I})\right] \hat{u}(0, k)=\mathbf{o},
$$

prohibits the non-decreasing modes in the solution of the corresponding homogeneous system as $x \longrightarrow-\infty$ and boundary condition

$$
\left[\prod_{\Re \lambda(\boldsymbol{k}) \geq 0}(\boldsymbol{Q}(\boldsymbol{k})-\lambda(\boldsymbol{k}) \boldsymbol{I})\right] \hat{\boldsymbol{u}}(X, \boldsymbol{k})=\mathbf{o}
$$

prohibits the modes that increase as $x \longrightarrow+\infty$. Therefore, boundary conditions (2.27) are equivalent to (2.22) in the sense that the solution to $(2.21),(2.27)$ on $[0, X]$ will be the same as the corresponding fragment of the solution given by (2.23). In formulas $(2.27), k \equiv\left(k_{y}, k_{z}\right)$, $\hat{\boldsymbol{u}}(\cdot, \boldsymbol{k}) \equiv \hat{\boldsymbol{u}}\left(\cdot, \eta_{k_{y}}, \zeta_{k_{z}}\right), \boldsymbol{Q}(\boldsymbol{k}) \equiv \boldsymbol{C}^{-1}\left(i \eta_{k_{y}} \boldsymbol{D}+i \zeta_{k_{z}} \boldsymbol{E}-\eta_{k_{y}}^{2} \boldsymbol{F}-\zeta_{k_{z}}^{2} \boldsymbol{H}-\eta_{k_{y}} \zeta_{k_{z}} \boldsymbol{J}\right), \lambda(\boldsymbol{k})$ are the eigenvalues of $\boldsymbol{Q}(\boldsymbol{k})$, and $\boldsymbol{I}$ is the identity matrix of the appropriate dimension.

The formulation of the finite-domain AP is therefore complete. For a given compactly supported RHS $f, \operatorname{supp} f \subseteq D_{i n}$, it consists of solving system (2.16) on the parallelepiped $[0, X] \times[-Y / 2, Y / 2] \times[-Z / 2, Z / 2]$ with the periodicity boundary conditions in the $y$ and $z$ directions and boundary conditions (2.27) in the $x$ direction. As mentioned above, by increasing the periods $Y$ and $Z$ one can make the solution to this AP arbitrarily close to the original nonperiodic solution on any finite fixed neighborhood of $D_{i n}$.

We will designate the Green's, i.e., inverse, operator of the finite-domain AP by $G$ so that if $\boldsymbol{L} \boldsymbol{u}=\boldsymbol{f}$ then $\boldsymbol{u}=\boldsymbol{G} \boldsymbol{f}$. We also introduce the space $\mathcal{F} \ni \boldsymbol{f}$ of the RHS's for the finite-domain AP $\left(\forall \boldsymbol{f}: \operatorname{supp} \boldsymbol{f} \subseteq D_{i n}\right)$ and the space $\mathcal{U} \ni \boldsymbol{u}$ of its solutions so that $\boldsymbol{L}: \mathcal{U} \longmapsto \mathcal{F}$ and $\boldsymbol{G}: \mathcal{F} \longmapsto \mathcal{U}$. Keeping in mind that the functions $\boldsymbol{u} \in \mathcal{U}$ approximate the solutions to the infinite-domain AP of Section 2.3.1 in the sense mentioned above, we will henceforth consider those $\boldsymbol{u} \in \mathcal{U}$ as satisfying the appropriate boundary conditions at infinity.

2.3.3. Generalized Potentials and Boundary Projections. Let us now introduce the space of clear traces $\Xi$. The elements $\xi \in \Xi$ are the vector-functions defined on the artificial boundary $\Gamma$; typically, for any $\boldsymbol{u} \in \mathcal{U}$ we may consider $\xi=\left.\left(\boldsymbol{u}, \frac{\partial \boldsymbol{u}}{\partial n}\right)\right|_{\Gamma}$ where $n$ is the normal to $\Gamma$. The concept of clear trace is delineated in $[6,7]$. The operator $\boldsymbol{T r}: \mathcal{U} \longmapsto \Xi$ that associates the clear trace with each $\boldsymbol{u} \in \mathcal{U}$ is called the clear trace operator.

Let now some $\xi \in \Xi$ be prescribed. One can always find a compactly supported function $v$ such that $\boldsymbol{T} \boldsymbol{r} \boldsymbol{v}=\boldsymbol{\xi}$. Then, the truncated function $\boldsymbol{f}=\left.(\boldsymbol{L} \boldsymbol{v})\right|_{D_{i n}} \in \mathcal{F}$ can be a RHS for the finite-domain AP. The corresponding solution of the finite-domain AP considered only on $D_{e x}$ is called the generalized potential with the density $\xi:\left.\boldsymbol{P} \xi \stackrel{\text { def }}{=}\left[\boldsymbol{G}\left(\left.(\boldsymbol{L} \boldsymbol{v})\right|_{D_{i n}}\right)\right]\right|_{D_{e x}}$. The generalized potential can be shown to depend only on its density $\xi$ and not on the choice of $\boldsymbol{v}$ (see $[6,7]$ ).

The composition of operators $\boldsymbol{P}$ and $\boldsymbol{T r}, \boldsymbol{P}_{\Gamma} \stackrel{\text { def }}{=} \boldsymbol{T r} \boldsymbol{P}$, maps the space of clear traces onto itself, $\boldsymbol{P}_{\Gamma}: \Xi \longmapsto \Xi$. This new operator is a projection, $\boldsymbol{P}_{\Gamma}^{2}=\boldsymbol{P}_{\Gamma}$, and is called the generalized boundary projection. Those and only those $\xi \in \Xi$ that belong to the image of the generalized boundary projection, $\xi \in \operatorname{Im} \boldsymbol{P}_{\Gamma}$, or in other words, satisfy the boundary equation with projection $\xi=\boldsymbol{P}_{\Gamma} \xi$, are actually the traces of some $\boldsymbol{u} \in \mathcal{U}$.

In the next section, we construct the finite-difference counterparts to the generalized potentials and boundary projections and apply those to setting the ABC's. 


\subsection{Computation of the DPM-based ABC's.}

2.4.1. Formulation of the Difference AP. Let us introduce a Cartesian grid on the parallelepiped $[0, X] \times[-Y / 2, Y / 2] \times[0, Z / 2] \supset \Gamma_{1}$. By virtue of symmetry (see Section 2.2 and, in particular, formulas $(2.14),(2.15)$ ), we may consider only a half of the domain along the coordinate $z$. The $x$-grid is uniform with the size $h_{x}: x_{m}=m h_{x}, m=0,1, \ldots, M, x_{0}=0, x_{M}=X$. The grids in $y$ and $z$ can also be uniform with the sizes $h_{y}$ and $h_{z}$, respectively: $y_{j_{y}}=-Y / 2+j_{y} h_{y}$, $j_{y}=0,1, \ldots, 2 J_{y}+1, y_{0}=-Y / 2, y_{2 J_{y}+1}=Y / 2$, and $z_{j_{z}}=-Z / 2+j_{z} h_{z}, j_{z}=J_{z}, \ldots, 2 J_{z}+1$, $z J_{z}=-h_{z} / 2, z_{2 J_{z}+1}=Z / 2$. (For the $z$-grid, we use here the same indexing of nodes as if it would be if we considered the entire interval $[-Z / 2, Z / 2]$ rather than only its half $[0, Z / 2]$. This is done mostly to keep consistency in the notations.) However, as we expect to have better accuracy for bigger periods $Y$ and $Z$ (see Section 2.3.2), it may be convenient for applications to keep the $y$ and $z$-grids uniform only in the vicinity of $D_{i n}$ and then stretch them away from the computational domain. This will allow us to cover bigger periods with the same number of nodes. In so doing, we can retain the same indexing for the nodes $y_{i_{y}}$ and $z_{j_{z}}$ but the grid sizes $h_{y}$ and $h_{z}$ will no longer be constant. In all our computations (Section 3), we have actually used the stretched grids in the $y$ and $z$ directions.

We designate the entire three-dimensional Cartesian grid by $\mathrm{N}^{0}, \mathrm{~N}^{0} \equiv\left\{\left(x_{m}, y_{j_{y}}, z_{j_{z}}\right) \mid m=0,1, \ldots, M, j_{y}=0,1, \ldots, 2 J_{y}+1, j_{z}=J_{z}, \ldots, 2 J_{z}+1\right\}$. The solutions $\boldsymbol{u}^{h} \in \mathcal{U}^{h}$ of the difference AP will be defined on this grid. We also introduce another Cartesian grid $\mathrm{M}^{0}$, on which we will define the RHS's $f^{h} \in \mathcal{F}^{h}$ of the difference AP. Compared to the nodes of the grid $\mathrm{N}^{0}$, the nodes of the new grid $\mathrm{M}^{0}$ are shifted half-size in $x: \mathrm{M}^{0} \equiv\left\{\left(x_{m-1 / 2}, y_{j_{y}}, z_{j_{z}}\right) \mid m=1, \ldots, M, j_{y}=0,1, \ldots, 2 J_{y}+1, j_{z}=J_{z}, \ldots, 2 J_{z}+1\right\}$, where $x_{m-1 / 2} \equiv(m-1 / 2) h_{x}$.

We discretize the operator $L$ of $(2.1)$ on the grid $\mathrm{N}^{0}$ with the second order of accuracy. The finite-difference scheme is centered with respect to the nodes $\left(m-1 / 2, j_{y}, j_{z}\right)$. To discretize $\frac{\partial u}{\partial x}$, we use the first-order differences in $x$, this ensures the second order of approximation because the residuals are evaluated on the same semi-integer grid $\mathrm{M}^{0}$, on which the RHS's are specified. For the first derivatives $\frac{\partial u}{\partial y}$ and $\frac{\partial u}{\partial z}$, we use the three-point second-order discretization and designate the corresponding grid operators by $\boldsymbol{D}_{y}$ and $\boldsymbol{D}_{z}$, respectively. The dimension of this operators

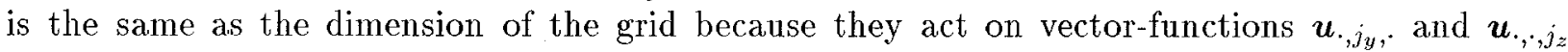
componentwise. On the uniform grid, this discretization turns into the standard central differencing as the central node drops out, but if the grid is stretched the discretization contains all three nonzero coefficients. The second derivatives $\frac{\partial^{2} \boldsymbol{u}}{\partial y^{2}}, \frac{\partial^{2} u}{\partial z^{2}}$, and $\frac{\partial^{2} \boldsymbol{u}}{\partial y \partial z}$ are discretized by the appropriate compositions of the first difference derivatives; $D_{y}^{2}, D_{z}^{2}$, and $\boldsymbol{D}_{y} \boldsymbol{D}_{z}$, respectively. We will designate the discrete direct operator by $L^{h}$.

Let now $\boldsymbol{u}^{h} \equiv \boldsymbol{u}_{m, j_{y}, j_{z}}$ and $\boldsymbol{f}^{h} \equiv \boldsymbol{f}_{m-1 / 2, j_{y}, j_{z}}$. Because of the periodicity in $y$,

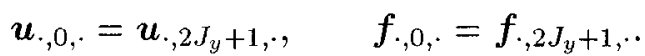

Also, because of the symmetry/antisymmetry with respect to $z=0$ (see boundary conditions $(2.14),(2.15))$ and periodicity in $z$,

$$
\begin{aligned}
& u_{,,, j z}^{i}=u_{\cdot,, 2 J_{z}+1-j_{z}}^{i}, \quad f_{{ }^{\prime}, j_{z}}^{i}=f_{\cdot, \cdot, 2 J_{z}+1-j_{z}}^{i}, \quad j_{z}=0,1, \ldots, J_{z}, \quad \text { for } i \neq 4, \\
& u_{\cdot,, j_{z}}^{i}=-u_{\cdot, \cdot, 2 J_{z}+1-j_{z}}^{i}, \quad f_{\cdot,,, j_{z}}^{i}=-f_{\cdot, \cdot, 2 J_{z}+1-j_{z}}^{i}, j_{z}=0,1, \ldots, J_{z}, \quad \text { for } i=4 \text {. }
\end{aligned}
$$

Again, whereas we formally enumerate the $z$-nodes from 0 to $2 J_{z}+1$ in $(2.28 \mathrm{~b})$, this formulas in fact show how one can consider only half of these nodes instead. 
To replace the continuous transforms (2.20), we introduce the discrete transforms $\boldsymbol{T}^{(y)}$ and $T^{(z)}$ so that for each $i$ :

$$
\begin{aligned}
& \hat{u}_{\cdot, k_{y}, k_{z}}^{i}=\sum_{j_{z}=1}^{j_{z}=2 J_{z}+1} \sum_{j_{y}=1}^{j_{y}=2 J_{y}+1} T_{k_{z}, j_{z}}^{(z)} T_{k_{y}, j_{y}}^{(y)} u_{\cdot, j_{y}, j_{z}}^{i}, \\
& \hat{f}_{,_{,} k_{y}, k_{z}}=\sum_{j_{z}=1}^{j_{z}=2 J_{z}+1} \sum_{j_{y}=1}^{j_{y}=2 J_{y}+1} T_{k_{z}, j_{z}}^{(z)} T_{k_{y}, j_{y}}^{(y)} f_{\cdot, j_{y}, j_{z} \cdot}^{i} .
\end{aligned}
$$

The operators $T^{(y)}$ and $T^{(z)}$ have the inverse that we denote by $\tilde{T}^{(y)} \equiv T^{(y)-1}$ and $\tilde{T}^{(z)} \equiv T^{(z)^{-1}}$, respectively, so that

$$
\begin{gathered}
u_{\cdot, j_{y}, j_{z}}^{i}=\sum_{k_{z}=-J_{z}}^{k_{z}=J_{z}} \sum_{k_{y}=-J_{y}}^{k_{y}=J_{y}} \tilde{T}_{j_{z}, k_{z}}^{(z)} \tilde{T}_{j_{y}, k_{y}}^{(y)} \hat{u}_{\cdot, k_{y}, k_{z}}^{i}, \\
f_{\cdot, j_{y}, j_{z}}^{i}=\sum_{k_{z}=-J_{z}}^{k_{z}=J_{z}} \sum_{k_{y}=-J_{y}}^{k_{y}=J_{y}} \tilde{T}_{j_{z}, k_{z}}^{(z)} \tilde{T}_{j_{y}, k_{y}}^{(y)} \hat{f}_{\cdot, k_{y}, k_{z}}^{i} .
\end{gathered}
$$

We require that the operator $T^{(y)}$ diagonalize the first and consequently, the second difference derivative with respect to $y$ :

$$
\tilde{\boldsymbol{T}}^{(y)} \boldsymbol{D}_{y} \boldsymbol{T}^{(y)}=\operatorname{diag}\left\{i \eta_{k_{y}}\right\}, \quad \tilde{\boldsymbol{T}}^{(y)} \boldsymbol{D}_{y}^{2} \boldsymbol{T}^{(y)}=\operatorname{diag}\left\{-\eta_{k_{y}}^{2}\right\},
$$

where $\eta_{k_{y}}, k_{y}=-J_{y}, \ldots, J_{y}$, are real. Similarly, we require that the operator $\boldsymbol{T}^{(y)}$ diagonalize the first and the second difference derivatives with respect to $z$ :

$$
\tilde{\boldsymbol{T}}^{(z)} \boldsymbol{D}_{z} \boldsymbol{T}^{(z)}=\operatorname{diag}\left\{i \zeta_{k_{z}}\right\}, \quad \tilde{\boldsymbol{T}}^{(z)} \boldsymbol{D}_{z}^{2} \boldsymbol{T}^{(z)}=\operatorname{diag}\left\{-\zeta_{k_{z}}^{2}\right\}
$$

where $\zeta_{k_{y}}, k_{z}=-J_{z}, \ldots, J_{z}$, are also real. From (2.31a) and (2.31b) it follows that

$$
\tilde{\boldsymbol{T}}^{(z)} \tilde{\boldsymbol{T}}^{(y)} \boldsymbol{D}_{y} \boldsymbol{D}_{z} \boldsymbol{T}^{(y)} \boldsymbol{T}^{(z)}=-\operatorname{diag}\left\{\eta_{k_{y}}\right\} \operatorname{diag}\left\{\zeta_{k_{z}}\right\}
$$

Clearly, the columns of the matrix $\boldsymbol{T}^{(y)}$ should therefore be the eigenvectors of $\boldsymbol{D}_{y}$ and analogously, the columns of $\boldsymbol{T}^{(z)}$ should be the eigenvectors of $\boldsymbol{D}_{z}$.

Note, in practical computations on the stretched grids (Section 3) the eigenvectors and eigenvalues of $\boldsymbol{D}_{y}$ and $\boldsymbol{D}_{z}$ are calculated with the standard IMSL subroutines. Although the resulting bases are, generally speaking, not orthogonal, the accuracy provided by this calculations is high. In fact, this accuracy far exceeds any requirements to the accuracy of ABC's that may originate from the accuracy of the interior solver. The inverse operators $\tilde{T}^{(y)} \equiv T^{(y)^{-1}}$ and $\tilde{T}^{(z)} \equiv T^{(z)^{-1}}$ are also found with the help of the standard IMSL subroutines.

If, in particular, the grids in $y$ and $z$ are uniform, then $\boldsymbol{T}^{(y)}$ and $\boldsymbol{T}^{(z)}$ are reduced to the well-known discrete Fourier transforms (from here on, the overbar - means complex conjugate): 


$$
T_{k_{y}, j_{y}}^{(y)}=\frac{e^{-i k_{y} j_{y} h_{y} \frac{2 \pi}{Y}}}{\sqrt{2 J_{y}+1} \sqrt{2 J_{z}+1}}, \quad T_{k_{z}, j_{z}}^{(z)}=\frac{e^{-i k_{z} j_{z} h_{z} \frac{2 \pi}{Z}}}{\sqrt{2 J_{y}+1} \sqrt{2 J_{z}+1}}
$$

$$
\tilde{T}_{j_{y}, k_{y}}^{(y)}=\bar{T}_{k_{y}, j_{y}}^{(y)}=\frac{e^{i k_{y} j_{y} h_{y} \frac{2 \pi}{Y}}}{\sqrt{2 J_{y}+1} \sqrt{2 J_{z}+1}}, \quad \tilde{T}_{j_{z}, k_{z}}^{(z)}=\bar{T}_{k_{z}, j_{z}}^{(z)}=\frac{e^{i k_{z} j_{z} h_{z} \frac{2 \pi}{Z}}}{\sqrt{2 J_{y}+1} \sqrt{2 J_{z}+1}}
$$

Let us now consider a special class of grids, namely, $y_{j_{y}}=-y_{2 J_{y}+1-j_{y}}$ for $j_{y}=0, \ldots, J_{y}$ and $z_{j_{z}}=-z_{2 J_{z}+1-j_{z}}$ for $j_{z}=0, \ldots, J_{z}$. Obviously, all uniform grids belong to this class, for the stretched grids it means symmetric stretching. Then, one can make sure that

$$
\begin{gathered}
T_{-k_{y}, j_{y}}^{(y)}=\bar{T}_{k_{y}, j_{y}}^{(y)} \text { for } k_{y}=0, \ldots, J_{y}, \\
T_{-k_{z}, j_{z}}^{(z)}=\bar{T}_{k_{z}, j_{z}}^{(z)} \text { for } k_{z}=0, \ldots, J_{z}, \\
T_{k_{y}, 2 J_{y}+1-j_{y}}^{(y)}=\bar{T}_{k_{y}, j_{y}}^{(y)} \text { for } j_{y}=0, \ldots, J_{y}, \\
T_{k_{z}, 2 J_{z}+1-j_{z}}^{(z)}=\bar{T}_{k_{z}, j_{z}}^{(z)} \text { for } \quad j_{z}=0, \ldots, J_{z},
\end{gathered}
$$

and also

$$
\begin{gathered}
\tilde{T}_{j_{y}, k_{y}}^{(y)}=\overline{\tilde{T}}_{j_{y}, k_{y}}^{(y)} \text { for } \quad k_{y}=0, \ldots, J_{y}, \\
\tilde{T}_{j_{z},-k_{z}}^{(z)}=\overline{\tilde{T}}_{j_{z}, k_{z}}^{(z)} \text { for } \quad k_{z}=0, \ldots, J_{z}, \\
\tilde{T}_{2 J_{y}+1-j_{y}, k_{y}}^{(y)}=\overline{\tilde{T}}_{j_{y}, k_{y}}^{(y)} \text { for } j_{y}=0, \ldots, J_{y}, \\
\tilde{T}_{2 J_{z}+1-j_{z}, k_{z}}^{(z)}=\overline{\tilde{T}}_{j_{z}, k_{z}}^{(z)} \text { for } j_{z}=0, \ldots, J_{z} .
\end{gathered}
$$

For the discrete Fourier transform on uniform grids, relations (2.33) and (2.34) immediately follow from (2.32a) and (2.32b), respectively; for the nonuniform grids these relations are verified experimentally.

Substituting (2.33) into (2.29b), taking into account relations $(2.28)$ and also that $\boldsymbol{f}^{h}$ is real, we obtain for $i \neq 4(\Re$ means the real part):

$$
\begin{gathered}
\text { (2.35a) } \hat{f}_{\cdot,\left|k_{y}\right|,\left|k_{z}\right|}^{i}=\sum_{j_{y}=1}^{j_{y}=2 J_{y}+1}\left[\sum_{j_{z}=J_{z}+1}^{j j_{z}=2 J_{z}}\left(2 T_{\left|k_{y}\right|, j_{y}}^{(y)} \Re T_{\left|k_{z}\right|, j_{z}}^{(z)} f_{\cdot, j_{y}, j_{z}}^{i}\right)+T_{\left|k_{y}\right|, j_{y}}^{(y)} \Re T_{\left|k_{z}\right|, 2 J_{z}+1}^{(z)} f_{\cdot, j_{y}, 2 J_{z}+1}^{i}\right], \\
\hat{f}_{\cdot,\left|k_{y}\right|,-\left|k_{z}\right|}^{i}=\hat{f}_{\cdot,\left|k_{y}\right|,\left|k_{z}\right|}^{i}, \quad \hat{f}_{\cdot,-\left|k_{y}\right|,\left|k_{z}\right|}^{i}=\hat{f}_{\cdot,-\left|k_{y}\right|,-\left|k_{z}\right|}^{i}=\overline{\hat{f}}_{\cdot,\left|k_{y}\right|,\left|k_{z}\right|}^{i},
\end{gathered}
$$

and for $i=4$ ( $\Im$ means the imaginary part):

$$
\begin{aligned}
(2.35 \mathrm{~b}) \hat{f}_{\cdot,\left|k_{y}\right|,\left|k_{z}\right|}^{i}= & i \sum_{j_{y}=1}^{j_{y}=2 J_{y}+1} \sum_{j_{z}=J_{z}+1}^{j_{z}=2 J_{z}} 2 T_{\left|k_{y}\right|, j_{y}}^{(y)} \Im T_{\left|k_{z}\right|, j_{z}}^{(z)} f_{\cdot, j_{y}, j_{z}}^{i} \\
& \hat{f}_{\cdot,\left|k_{y}\right|,-\left|k_{z}\right|}^{i}=-\hat{f}_{\cdot,\left|k_{y}\right|,\left|k_{z}\right|}^{i}, \hat{f}_{\cdot,-\left|k_{y}\right|,\left|k_{z}\right|}^{i}=-\overline{\hat{f}}_{\cdot,\left|k_{y}\right|,\left|k_{z}\right|}^{i}, \hat{f}_{\cdot,-\left|k_{y}\right|,-\left|k_{z}\right|}^{i}=\overline{\hat{f}}_{\cdot,\left|k_{y}\right|,\left|k_{z}\right|}^{i} .
\end{aligned}
$$


The relations similar to (2.35) can also be obtained for $\boldsymbol{u}^{h}$ on the basis of formulas $(2.28),(2.29 \mathrm{a})$, and (2.33). Furthermore, taking into account that $\boldsymbol{u}^{h}$ is real and using formulas (2.30a) and (2.34) we obtain for the inverse transform if $i \neq 4$ :

$$
\begin{aligned}
u_{\cdot, j_{y}, j_{z}}^{i}= & 4 \sum_{k_{z}=1}^{k_{z}=J_{z}} \sum_{k_{y}=1}^{k_{y}=J_{y}}\left(\Re \tilde{T}_{j_{z}, k_{z}}^{(z)} \Re \tilde{T}_{j_{y}, k_{y}}^{(y)} \Re \hat{u}_{\cdot, k_{y}, k_{z}}^{i}-\Re \tilde{T}_{j_{z}, k_{z}}^{(z)} \Im \tilde{T}_{j_{y}, k_{y}}^{(y)} \Im \hat{u}_{\cdot, k_{y}, k_{z}}^{i}\right)+ \\
& 2 \sum_{k_{z}=1}^{k_{z}=J_{z}} \Re \tilde{T}_{j_{z}, k_{z}}^{(z)} \Re \tilde{T}_{j_{y}, 0}^{(y)} \Re \hat{u}_{\cdot, 0, k_{z}}^{i}+ \\
& 2 \sum_{k_{y}=1}^{k_{y}=J_{y}}\left(\Re \tilde{T}_{j_{z}, 0}^{(z)} \Re \tilde{T}_{j_{y}, k_{y}}^{(y)} \Re \hat{u}_{\cdot, k_{y}, 0}^{i}-\Re \tilde{T}_{j_{z}, 0}^{(z)} \Im \tilde{T}_{j_{y}, k_{y}}^{(y)} \Im \hat{u}_{\cdot, k_{y}, 0}^{i}\right)+ \\
& T_{j_{z}, \mathbf{0}}^{(z)} T_{j_{y}, 0}^{(y)} \hat{u}_{\cdot, \mathbf{0}, 0}^{i}
\end{aligned}
$$

and if $i=4$ :

$$
\begin{aligned}
u_{\cdot, j_{y}, j_{z}}^{i}= & -4 \sum_{k_{z}=1}^{k_{z}=J_{z}} \sum_{k_{y}=1}^{k_{y}=J_{y}}\left(\Im \tilde{T}_{j_{z}, k_{z}}^{(z)} \Re \tilde{T}_{j_{y}, k_{y}}^{(y)} \Im \hat{u}_{\cdot, k_{y}, k_{z}}^{i}+\Im \tilde{T}_{j_{z}, k_{z}}^{(z)} \Im \tilde{T}_{j_{y}, k_{y}}^{(y)} \Re \hat{u}_{\cdot, k_{y}, k_{z}}^{i}\right) \\
& -2 \sum_{k_{z}=1}^{k_{z}=J_{z}} \Im \tilde{T}_{j_{z}, k_{z}}^{(z)} \Re \tilde{T}_{j_{y}, 0}^{(y)} \Im \hat{u}_{\cdot, 0, k_{z}}^{i} .
\end{aligned}
$$

Usage of the transforms (2.35) and (2.36) instead of (2.29) and (2.30), respectively, allows us to calculate only one fourth out of the total number of coefficients, namely, those for $k_{y}=0,1, \ldots, J_{y}$ and $k_{z}=0,1, \ldots, J_{z}$. This obviously implies a four-fold speedup and four-fold shrinkage of the storage requirements when implementing in practice the separation of variables for the difference AP.

In the transformed space, instead of $\boldsymbol{L}^{h} \boldsymbol{u}^{h}=\boldsymbol{f}^{h}$ we obtain a family of one-dimensional systems:

$$
\begin{gathered}
\boldsymbol{A}_{\boldsymbol{k}} \hat{\boldsymbol{u}}_{m, \boldsymbol{k}}+\boldsymbol{B}_{\boldsymbol{k}} \hat{\boldsymbol{u}}_{m-1, \boldsymbol{k}}=\hat{\boldsymbol{f}}_{m-1 / 2, \boldsymbol{k}}, \\
m=1, \ldots, M, \boldsymbol{k} \equiv\left(k_{y}, k_{z}\right) \\
k_{y}=0, \ldots, J_{y}, k_{z}=0, \ldots, J_{z}
\end{gathered}
$$

where

$$
\begin{gathered}
\boldsymbol{A}_{\boldsymbol{k}}=\frac{1}{h_{x}} \boldsymbol{C}+\frac{i \eta_{k_{y}}}{2} \boldsymbol{D}+\frac{i \zeta_{k_{z}}}{2} \boldsymbol{E}-\frac{\eta_{k_{y}}^{2}}{2} \boldsymbol{F}-\frac{\zeta_{k_{z}}^{2}}{2} \boldsymbol{H}-\frac{\eta_{k_{y}} \zeta_{k_{z}}}{2} \boldsymbol{J} \\
\boldsymbol{B}_{\boldsymbol{k}}=-\frac{1}{h_{x}} \boldsymbol{C}+\frac{i \eta_{k_{y}}}{2} \boldsymbol{D}+\frac{i \zeta_{k_{z}}}{2} \boldsymbol{E}-\frac{\eta_{k_{y}}^{2}}{2} \boldsymbol{F}-\frac{\zeta_{k_{z}}^{2}}{2} \boldsymbol{H}-\frac{\eta_{k_{y}} \zeta_{k_{z}}}{2} \boldsymbol{J}
\end{gathered}
$$

For each system (2.37), we have to specify the boundary conditions at $m=0$ and $m=M$. Analogously to the continuous boundary conditions (2.27), the boundary conditions for the discrete system should explicitly prohibit the corresponding growing modes of the solution. This can be achieved by setting 


$$
\left[\prod_{|\lambda(\boldsymbol{k})|>1}\left(\boldsymbol{Q}_{\boldsymbol{k}}-\lambda(\boldsymbol{k}) \boldsymbol{I}\right)\right] \hat{\boldsymbol{u}}_{0, k}=\mathbf{o}
$$

and

$$
\left[\prod_{|\lambda(k)| \leq 1}\left(\boldsymbol{Q}_{k}-\lambda(\boldsymbol{k}) \boldsymbol{I}\right)\right] \hat{\boldsymbol{u}}_{M, \boldsymbol{k}}=\mathbf{o}
$$

where $\boldsymbol{Q}_{\boldsymbol{k}}=\boldsymbol{A}_{\boldsymbol{k}}^{-1} \boldsymbol{B}_{\boldsymbol{k}}, \lambda(\boldsymbol{k})$ are the eigenvalues of $\boldsymbol{Q}_{\boldsymbol{k}}$, and $\boldsymbol{I}$ is the identity matrix of the appropriate dimension.

The finite-difference AP has thus been formulated completely. It consists of solving the discrete system $\boldsymbol{L}^{h} \boldsymbol{u}^{h}=\boldsymbol{f}^{h}$ on the grid $\mathrm{N}^{0}$ with the RHS specified on the grid $\mathrm{M}^{0}$. The boundary conditions in the directions $y$ and $z$ are periodicity and symmetry, see (2.28). The boundary conditions in the direction $x$ are specified by formulas (2.39) in the transformed space separately for each component after the original system $\boldsymbol{L}^{h} \boldsymbol{u}^{h}=\boldsymbol{f}^{h}$ has been reduced to (2.37), (2.38) by the separation of variables (2.35), (2.36). The methodology for solving systems (2.37), (2.38) with boundary conditions (2.39), as well as the specific structure of these boundary conditions, are studied in the next section.

2.4.2. Solvability of the Difference AP. Let us first concentrate here on the incompressible case, when the $4 \times 4$ system matrices are given in (2.1b). For simplicity, we will temporarily omit the indices $\boldsymbol{k}$. If $\eta \equiv \eta_{k_{y}} \neq 0$ or $\zeta \equiv \zeta_{k_{z}} \neq 0$, then the solutions $\lambda_{s} \equiv \lambda_{s}(\boldsymbol{k})$ and $\boldsymbol{e}_{s} \equiv \boldsymbol{e}_{s}(\boldsymbol{k})$, $s=1, \ldots, 4$, of the problem $\boldsymbol{B}_{k} \boldsymbol{e}-\lambda \boldsymbol{A}_{\boldsymbol{k}}=\mathbf{o}$ are given by

$$
\begin{aligned}
& \lambda_{1}=-\left(\frac{1}{h_{x}}-\frac{\eta^{2}+\zeta^{2}}{2 R e}\right)\left(\frac{1}{h_{x}}+\frac{\eta^{2}+\zeta^{2}}{2 R e}\right)^{-1}=\lambda_{2} \\
& \lambda_{3}=\left(\frac{\sqrt{\eta^{2}+\zeta^{2}}}{2}-\frac{1}{h_{x}}\right)\left(\frac{\sqrt{\eta^{2}+\zeta^{2}}}{2}+\frac{1}{h_{x}}\right)^{-1} \\
& \lambda_{4}=\left(\frac{\sqrt{\eta^{2}+\zeta^{2}}}{2}+\frac{1}{h_{x}}\right)\left(\frac{\sqrt{\eta^{2}+\zeta^{2}}}{2}-\frac{1}{h_{x}}\right)^{-1} \\
& \boldsymbol{e}_{1}=[0,0,-\zeta, \eta]^{t} \\
& \boldsymbol{e}_{2}=\left[0,1, \frac{-i \eta}{R e}, \frac{-i \zeta}{\operatorname{Re}}\right]^{t} \\
& \boldsymbol{e}_{3}=\left[-\sqrt{\eta^{2}+\zeta^{2}}+\frac{\eta^{2}+\zeta^{2}}{R e}, \sqrt{\eta^{2}+\zeta^{2}},-i \eta,-i \zeta\right]^{t} \\
& \boldsymbol{e}_{4}=\left[\sqrt{\eta^{2}+\zeta^{2}}+\frac{\eta^{2}+\zeta^{2}}{R e},-\sqrt{\eta^{2}+\zeta^{2}},-i \eta,-i \zeta\right]^{t}
\end{aligned}
$$

From (2.40) we see that we have to analyze two different cases. In the regular case when $\sqrt{\eta^{2}+\zeta^{2}} / 2-1 / h_{x} \neq 0$, none of the eigenvalues $\lambda_{s}$ degenerate, the inverse $\boldsymbol{A}_{\boldsymbol{k}}^{-1}$ exists, and the eigenvalues/eigenvectors (2.40) are also the eigenvalues/eigenvectors of $\boldsymbol{Q}_{\boldsymbol{k}}$. The determinant of the Gram matrix constructed on the normalized eigenvectors $e_{s}$ from $(2.40)$ can be shown to be 


$$
\operatorname{Det}_{G}=\frac{4\left(1-\frac{\eta^{2}+\zeta^{2}}{R e^{2}}\right)^{2}}{\left(1+\frac{\eta^{2}+\zeta^{2}}{R e^{2}}\right)\left(\left(1+\frac{\eta^{2}+\zeta^{2}}{R e^{2}}\right)^{2}+8\right)}
$$

Therefore, if $\left(\eta^{2}+\zeta^{2}\right) \neq R e^{2}$ then the eigenvectors $\boldsymbol{e}_{s}$ are linearly independent and for $\sqrt{\eta^{2}+\zeta^{2}} / 2-$ $1 / h_{x} \neq 0$ we can diagonalize the system (2.37):

$$
S_{k}^{-1} Q_{k} S_{k}=\operatorname{diag}\left\{\lambda_{s}\right\}, \quad \text { where } S_{k}=\left[\frac{e_{1}}{\left|e_{1}\right|}, \frac{e_{2}}{\left|e_{2}\right|}, \frac{e_{3}}{\left|e_{3}\right|}, \frac{e_{4}}{\left|e_{4}\right|}\right]
$$

Let us note that since $\eta^{2} \lesssim 1 / h_{y}^{2}$ and $\zeta^{2} \lesssim 1 / h_{z}^{2}\left(h_{y}\right.$ and $h_{z}$ are the smallest grid sizes) then the condition $\left(\eta^{2}+\zeta^{2}\right) \neq R e^{2}$ appears not too restrictive. For example, the asymptotic width of the plane viscous wake in the far field behind the body is $\sim 1 / \sqrt{R e}$ (see, e.g., [45]). Therefore, to resolve this structure it is sufficient to have the grid sizes of order $1 / \sqrt{R e}$ as well ( $R e$ is an effective turbulent Reynolds number), which puts the operator $\boldsymbol{S}_{\boldsymbol{k}}$ of (2.42) far away of the possible singularity. We also note that in the formal inviscid limit $1 / R e \longrightarrow 0$, the determinant Det $_{G}$ of the Gram matrix (see (2.41)) becomes fully independent of the wavenumbers $\eta$ and $\zeta$, which essentially means that the "extent of skewness" for the basis $\left\{\boldsymbol{e}_{s}\right\}$ will be constant.

The solution to the diagonalized system (2.37) is easy to find by marching those components, for which $\lambda_{s} \leq 1$, from left to right and those components, for which $\lambda_{s}>1$, from right to left. It is also easy to make sure that boundary conditions (2.39a) essentially imply that the components, for which $\lambda_{s}>1$, are not specified (i.e., can be arbitrary) at the left end of the interval and the components, for which $\lambda_{s} \leq 1$ (those that would not decay as $m \longrightarrow-\infty$ ), are zero at $m=0$. Similarly, boundary conditions $(2.39 \mathrm{~b})$ mean that the components, for which $\lambda_{s} \leq 1$, are not specified (i.e., any value is admitted) at the right end of the interval and the components, for which $\lambda_{s}>1$ (those that would increase as $m \longrightarrow+\infty$ ), are zero at $m=M$. Let us also note that $1 /$ Re may be arbitrarily small but as long as it is positive, $\left|\lambda_{s}\right| \neq 1$ for all $s$. Consequently, we have only growing and decaying modes and no constant or oscillating modes in the solution of the corresponding homogeneous system. Therefore, in accordance with the results of [46] we have arrived at

Proposition 2.6. Let $\eta_{k_{y}} \neq 0$ or $\zeta_{k_{z}} \neq 0$; let also $\sqrt{\eta_{k_{y}}^{2}+\zeta_{k_{z}}^{2}} / 2-1 / h_{x} \neq 0$. Then, system (2.37), (2.38), (2.16) with boundary conditions (2.39) is uniquely solvable and wellposed for any compactly supported $R H S \hat{f}_{m-1 / 2, k}$. The constant in the well-posedness estimate $\left\|\hat{u}_{\cdot, k}\right\| \leq$ const $\left\|\hat{f}_{\cdot, k}\right\|$ does not depend on $M$.

Note, the system $(2.37),(2.38),(2.1 \mathrm{~b}),(2.39)$ can also be solved using the methodology of [16].

The case $\sqrt{\eta^{2}+\zeta^{2}} / 2-1 / h_{x}=0$ requires special analysis. In this case, $\lambda_{3}=0$ and also formally $\lambda_{4}=\infty$. In fact, however, it is easy to make sure that both matrices $\boldsymbol{A}_{\boldsymbol{k}}$ and $\boldsymbol{B}_{\boldsymbol{k}}$ are singular for $\sqrt{\eta^{2}+\zeta^{2}} / 2-1 / h_{x}=0$. Let us therefore consider a regular pencil of matrices $\boldsymbol{A}_{\boldsymbol{k}}+\mu \boldsymbol{B}_{\boldsymbol{k}}$ (see, e.g., [47]). We can rewrite these pencil as follows: $\boldsymbol{A}_{\boldsymbol{k}}+\mu \boldsymbol{B}_{\boldsymbol{k}}=\left(\boldsymbol{A}_{\boldsymbol{k}}-\boldsymbol{B}_{\boldsymbol{k}}\right)+(\mu+1) \boldsymbol{B}_{\boldsymbol{k}} \equiv \boldsymbol{A}_{\boldsymbol{k}}^{\prime}+(\mu+1) \boldsymbol{B}_{k}$. As $\boldsymbol{A}_{\boldsymbol{k}}^{\prime}=\frac{2}{h_{x}} \boldsymbol{C}$, this matrix is non-singular and therefore $\boldsymbol{A}_{\boldsymbol{k}}+\mu \boldsymbol{B}_{\boldsymbol{k}}=\boldsymbol{A}_{\boldsymbol{k}}^{\prime}\left(\boldsymbol{I}+(\mu+1) \boldsymbol{A}_{\boldsymbol{k}}^{\prime-1} \boldsymbol{B}_{\boldsymbol{k}}\right)$. The combination of matrices in the brackets can be diagonalized, which yields: 


$$
\begin{aligned}
& \boldsymbol{A}_{\boldsymbol{k}}+\mu \boldsymbol{B}_{\boldsymbol{k}}= \\
& \boldsymbol{A}_{\boldsymbol{k}}^{\prime} \boldsymbol{S}_{\boldsymbol{k}}^{\prime}\left[\begin{array}{cccc}
1 & 0 & 0 & 0 \\
0 & \left(\frac{1}{2}+\frac{1}{h_{x} R e}\right)+\mu\left(-\frac{1}{2}+\frac{1}{h_{x} R e}\right) & 0 & 0 \\
0 & 0 & \left(\frac{1}{2}+\frac{1}{h_{x} R e}\right)+\mu\left(-\frac{1}{2}+\frac{1}{h_{x} R e}\right) & 0 \\
0 & 0 & 0 & -\mu
\end{array}\right] \boldsymbol{S}_{\boldsymbol{k}}^{\prime-1}
\end{aligned}
$$

where $S_{k}^{\prime}$ is the corresponding similarity transform. (It is easy to make sure that all the eigenvectors are linearly independent so that non-singular $\boldsymbol{S}_{\boldsymbol{k}}^{\prime}$ does exist.)

From representation (2.43) we conclude that there are still three components in the solution that should be calculated by marching from left to right and one component that should be calculated by marching from right to left. This obviously matches the structure of boundary conditions (2.39) as the latter can also be multiplied from the left by a nonsingular matrix $\boldsymbol{A}_{k}^{\prime}$. In fact, the pencil $\boldsymbol{A}_{\boldsymbol{k}}+\mu \boldsymbol{B}_{\boldsymbol{k}}$ has one zero elementary divisor that corresponds to marching from right to left, at least one "infinite" elementary divisor that corresponds to marching from left to right, and may have either two finite elementary divisors or another two "infinite" elementary divisors that would also correspond to marching from left to right. Clearly, any of these marching procedures will easily lead to an $M$-independent estimate of the resulting solution via the prescribed RHS. Therefore, we have justified

Proposition 2.7. Let $\eta_{k_{y}} \neq 0$ or $\zeta_{k_{z}} \neq 0$; let also $\sqrt{\eta_{k_{y}}^{2}+\zeta_{k_{z}}^{2}} / 2-1 / h_{x}=0$. Then, system (2.37), (2.38), (2.16) with boundary conditions (2.39) is uniquely solvable and wellposed for any compactly supported RHS $\hat{\boldsymbol{f}}_{m-1 / 2, k}$. The constant in the well-posedness estimate $\left\|\hat{\boldsymbol{u}}_{\cdot, \boldsymbol{k}}\right\| \leq \mathrm{const}\left\|\hat{f}_{\cdot, k}\right\|$ does not depend on $M$.

Let us now mention that for the discrete Fourier transforms on the uniform grids $\eta_{k_{y}}=$ $\sin \left(\frac{2 \pi k_{y} h_{y}}{Y}\right) / h_{y}$ and $\zeta_{k_{z}}=\sin \left(\frac{2 \pi k_{z} h_{z}}{Z}\right) / h_{z}$. Then, to avoid the considerations that result in proposition 2.7 and to restrict oneself by the case of proposition 2.6 only, one can impose the following limitation on the grid sizes: $h_{x}^{-2}>\left(h_{y}^{-2}+h_{z}^{-2}\right) / 4$. We also note that the general analysis of constantcoefficient ordinary difference equations based on the canonical forms of the corresponding pencils of matrices can be found in work [48].

The analysis of the last remaining case, $\eta_{k_{y}}=\zeta_{k_{y}}=0 \Longleftrightarrow \boldsymbol{k}=\mathbf{o}$, is straightforward as $\boldsymbol{Q}_{\mathrm{o}}=-\boldsymbol{I}$ and the solution of (2.37), (2.39) can therefore be found by marching all the components from left to right. In accordance with the results of [46] and [16], the well-posedness constant in this case is proportional to $M$.

For the compressible case (2.1c), the similar results also hold. However, the analytical expressions of type (2.40) are generally hard to obtain, so the actual eigenvalues and eigenvectors must be calculated numerically (we again use the standard IMSL subroutines). The critical value, for which the eigen-basis becomes singular (see proposition 2.7) is now $\sqrt{\eta_{k_{y}}^{2}+\zeta_{k_{z}}^{2}} / 2-\sqrt{1-M_{0}^{2}} / h_{x}=0$.

We will designate the Green's, i.e., inverse, operator of the difference AP by $G^{h}$ so that if $\boldsymbol{f}^{h} \in \mathcal{F}^{h}$ and $\boldsymbol{L}^{h} \boldsymbol{u}^{h}=\boldsymbol{f}^{h}$ then $\boldsymbol{u}^{h}=\boldsymbol{G}^{h} \boldsymbol{f}^{h}$ and $\boldsymbol{u}^{h} \in \mathcal{U}^{h}$.

2.4.3. Difference Potentials and Projections. Let $\mathrm{St}_{m-1 / 2, j_{y}, j_{z}}$ be the stencil of the difference operator $L^{h}$; according to Section 2.4.1, we use the first-order differences for the coordinate $x$ and the central-type differences and their products for the coordinates $y$ and $z$. Let us also introduce the following grid sets (the overbar $\bar{D}_{i n}$ here means the set-theoretical closure): 


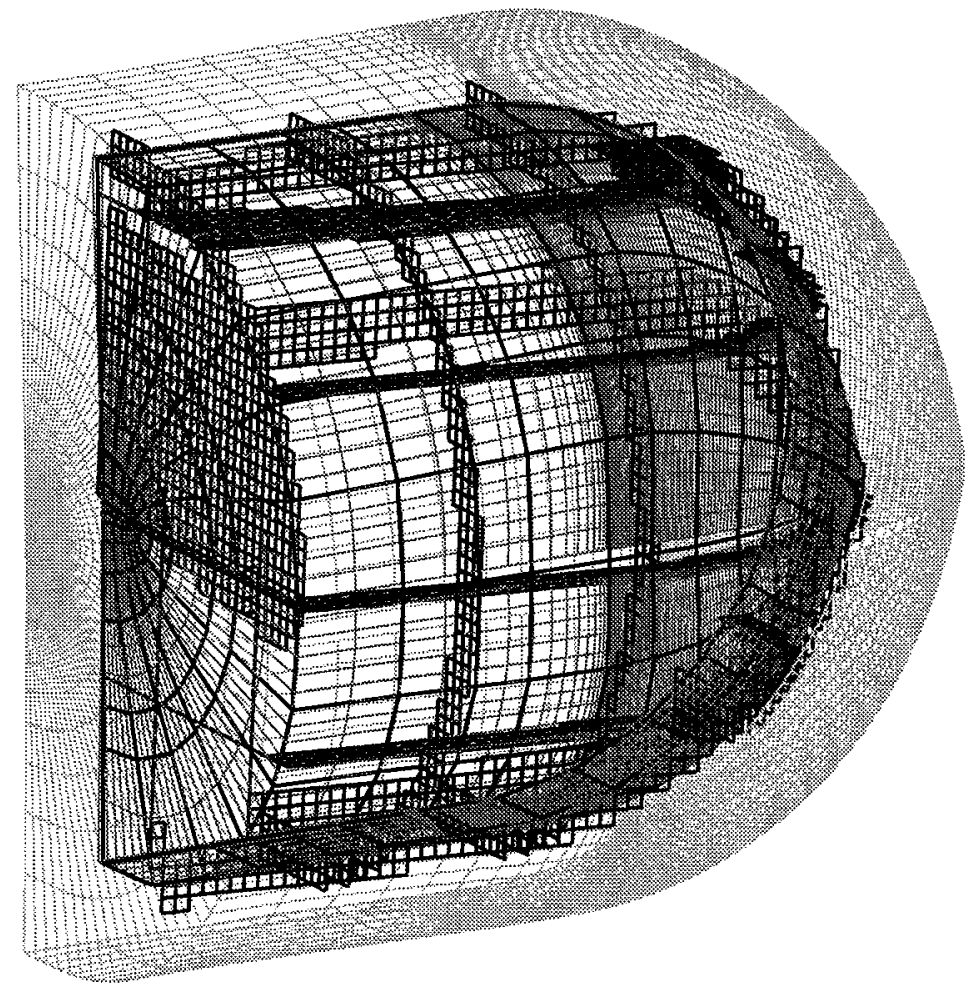

FIG. 2.2. Continuous artificial boundary $\Gamma$, grid boundary $\gamma$, collocation grid $\sigma$ on $\Gamma$, and ghost nodes $\Gamma_{1}$ for a typical three-dimensional configuration.

$$
\begin{gathered}
\mathrm{N}_{i n}=\bigcup_{\left(m-1 / 2, j_{y}, j_{z}\right) \in \mathrm{M}_{i n}} \mathrm{M}_{i n} \stackrel{\text { def }}{=} \mathrm{M}^{0} \bigcap \bar{D}_{i n}, \quad \mathrm{M}_{e x} \stackrel{\text { def }}{=} \mathrm{M}^{0} \bigcap D_{e x}, \\
\gamma=\mathrm{N}_{i n} \bigcap \mathrm{N}_{e x} .
\end{gathered}
$$

By definition (2.44), $\mathrm{M}_{i n}$ and $\mathrm{M}_{e x}$ do not have common nodes. The sets $\mathrm{N}_{\text {in }}$ and $\mathrm{N}_{e x}$ already have some common nodes because these sets are swept by the stencil $\mathrm{St}_{m-1 / 2, j_{y}, j_{z}}$ as it is applied to every node from $\mathrm{M}_{i n}$ and $\mathrm{M}_{e x}$, respectively. The intersection of $\mathrm{N}_{i n}$ and $\mathrm{N}_{e x}$ is called the grid boundary $\gamma$. It is actually a multi-layered fringe of nodes of the auxiliary Cartesian grid concentrated in the vicinity of the continuous artificial boundary $\Gamma$. Similarly to the continuous case (Section 2.3.3), the density of the generalized difference potential will be defined on the grid boundary $\gamma$. An example of the grid boundary (actually, a few planar cross-sections of this set) for a typical configuration studied in this paper is shown in Figure 2.2.

The difference clear traces $\xi_{\gamma} \in \Xi_{\gamma}$ of the functions $\boldsymbol{u}^{h} \in \mathcal{U}^{h}$ are now defined as merely the contractions to the grid boundary, i.e., $\left.\boldsymbol{T r}^{h} \boldsymbol{u}^{h} \stackrel{\text { def }}{=} \boldsymbol{u}^{h}\right|_{\gamma}=\xi_{\gamma}, \boldsymbol{T r}^{h}: \mathcal{U}^{h} \longmapsto \Xi_{\gamma}$.

Let now some $\xi_{\gamma} \in \Xi_{\gamma}$ be prescribed and $\boldsymbol{v}^{h}$ be a grid function defined on $\mathrm{N}^{0}$ such that $\boldsymbol{T} \boldsymbol{r}^{h} \boldsymbol{v}^{h}=\xi_{\gamma}$. Clearly, there are many functions $\boldsymbol{v}^{h}$ that would meet this condition, for example

$$
\boldsymbol{v}^{h}= \begin{cases}\xi_{\gamma} & \text { on } \gamma \\ \boldsymbol{o} & \text { on } \mathrm{N}^{0} \backslash \gamma\end{cases}
$$


Then, consider the function

$$
\mathcal{F}^{h} \ni f^{h}= \begin{cases}\boldsymbol{L}^{h} \boldsymbol{v}^{h} & \text { on } \mathrm{M}_{\text {in }}, \\ \boldsymbol{o} & \text { on } \mathrm{M}_{\text {ex }}\end{cases}
$$

where $\boldsymbol{v}^{h}$ is defined by (2.45), and solve the difference AP with this RHS $\boldsymbol{f}^{h}$ of (2.46). The resulting solution considered only on $\mathrm{N}_{e x}$ is called the generalized difference potential with the density $\xi_{\gamma}$ :

$$
\left.\boldsymbol{P}^{h} \xi_{\gamma} \stackrel{\text { def }}{=}\left(\boldsymbol{G}^{h} \boldsymbol{f}^{h}\right)\right|_{\mathrm{N}_{e x}}
$$

$\left(\boldsymbol{f}^{h}\right.$ in (2.47) is defined by (2.46)). Analogously to the continuous case (Section 2.3.3), the generalized potential $\boldsymbol{P}^{h} \xi_{\gamma}$ of (2.47) can be shown to depend only on its density $\xi_{\gamma}$ and not on the choice of $\boldsymbol{v}^{h}$ (if the latter differs from (2.45) but still $\boldsymbol{T} \boldsymbol{r}^{h} \boldsymbol{v}^{h}=\xi_{\gamma}$ ), see [7].

The composition of operators $\boldsymbol{P}^{h}$ and $\boldsymbol{T} \boldsymbol{r}^{h}, \boldsymbol{P}_{\gamma}^{h} \stackrel{\text { def }}{=} \boldsymbol{T} \boldsymbol{r}^{h} \boldsymbol{P}^{h}$, maps the space of the difference clear traces onto itself, $\boldsymbol{P}_{\gamma}^{h}: \Xi_{\gamma} \longmapsto \Xi_{\gamma}$. This new operator is a projection, $\boldsymbol{P}_{\gamma}^{h^{2}}=\boldsymbol{P}_{\Gamma}^{h}$, and is called the generalized difference boundary projection. Those and only those $\xi_{\gamma} \in \Xi_{\gamma}$ that belong to the image of the generalized difference boundary projection, $\xi_{\gamma} \in \operatorname{Im} \boldsymbol{P}_{\gamma}^{h}$, or in other words, satisfy the difference boundary equation with projection $\xi_{\gamma}=\boldsymbol{P}_{\gamma}^{h} \xi_{\gamma}$, are actually the traces of some $\boldsymbol{u}^{h} \in \mathcal{U}^{h}$.

Note, numerical verification of the projection property $\boldsymbol{P}_{\gamma}^{h^{2}}=\boldsymbol{P}_{\gamma}^{h}$ is an ideal test for accuracy of the solution of difference AP. In our practical computations for different geometries on different grids, we have always been able to obtain for arbitrary $\xi_{\gamma}$ 's: $\left\|\boldsymbol{P}_{\gamma}^{h^{2}} \xi_{\gamma}-\boldsymbol{P}_{\gamma}^{h} \xi_{\gamma}\right\|<10^{-9}$. This, in particular, justifies the usage of the stretched grids when solving the difference AP.

As mentioned before (Section 2.3.2), we consider the continuous functions $u \in \mathcal{U}$ as satisfying the appropriate boundary conditions at infinity because the difference between the non-periodic solution and its periodic approximation is controlled by $Y$ and $Z$ and can, in fact, be made as small as initially prescribed. The discrete space $\mathcal{U}^{h}$ approximates the continuous space $\mathcal{U}$, therefore we consider those grid densities $\xi_{\gamma}$ that belong to $\operatorname{Im} \boldsymbol{P}_{\gamma}^{h}, \xi_{\gamma} \in \operatorname{Im} \boldsymbol{P}_{\gamma}^{h}$ as admitting the exterior complement in the right sense. In other words, these and only these functions $\xi_{\gamma}$ admit such a complement $\boldsymbol{u}_{e x}^{h} \stackrel{\text { def }}{=} \boldsymbol{P}^{h} \xi_{\gamma}$ that satisfies the boundary conditions of the difference AP (see (2.28), (2.39)); this complement can therefore be made arbitrarily close (near $D_{i n}$ ) to an original linearized exterior solution; in the next section, it is used for setting the difference ABC's.

2.4.4. Global DPM-based Artificial Boundary Conditions. Having constructed the procedure for calculating the generalized difference potentials and projections, we can now provide for a closure to the discretized Navier-Stokes system that is solved inside the computational domain $D_{i n}$, i.e., obtain the ABC's. As mentioned in Section 2.2, the interior solvers typically involve some sort of pseudo-time iterations. To make every step of the iteration procedure, we need to know the previous-step solution everywhere on the grid, including the ghost nodes $\Gamma_{1}$. If these data are available, then on the next step we will know the solution everywhere except on $\Gamma_{1}$. Consequently, to advance another iteration we will have to supplement the missing data on $\Gamma_{1}$. This will be done by projecting the available boundary data at $\Gamma$ onto the "right manifold", i.e., the one that admits the right exterior complement (see the previous section), and then calculating this complement on $\Gamma_{1}$. In so doing, we can obtain the missing relations between the values of the solution on $\Gamma$ and $\Gamma_{1}$ every time the ghost nodes need to be updated.

First, let us introduce the intermediate collocation grids $\sigma$ and $\sigma_{1}$ on both surfaces $\Gamma$ and $\Gamma_{1}$. An example of such $\sigma \subset \Gamma$ is shown in Figure 2.2. These grids are typically a few times coarser than $\Gamma$ and $\Gamma_{1}$. Usage of the collocation grid on $\Gamma$ is an element of general procedure of the difference 
potentials method [7]. Moreover, for the specific problem under study usage of the collocation grids results in multi-fold acceleration of the computational procedure and also in memory savings if the boundary conditions are implemented in the matrix form (see below).

Then, let us take $\xi_{\Gamma}=\left.\left(\boldsymbol{u}, \frac{\partial \boldsymbol{u}}{\partial n}\right)\right|_{\Gamma}$ where $n$ is the normal to $\Gamma$ (these data are available from inside the computational domain $D_{i n}$ on every iteration) and, using the clustering $\boldsymbol{R}_{\sigma}$ on $\Gamma$, obtain $\xi_{\sigma}$. The latter procedure (clustering), in fact, implies local averaging or smoothening along $\Gamma$. Furthermore, we drop normals from all nodes $\gamma$ to the surface $\Gamma$ and interpolate $\xi_{\sigma}$ with sufficiently high order to the feet of these normals. The corresponding operation is denoted $\boldsymbol{R}_{n}$; typically, we use the bi-quadratic surface interpolation. Having obtained $\boldsymbol{u}$ and $\frac{\partial \boldsymbol{u}}{\partial n}$ at the feet of the normals, we use the first two terms of the Taylor expansion (denoted $\pi_{\gamma}$ ) and obtain $\xi_{\gamma}$ :

$$
\xi_{\gamma}=\left.\pi_{\gamma} \boldsymbol{R}_{n} \boldsymbol{R}_{\sigma}\left(u, \frac{\partial u}{\partial n}\right)\right|_{\Gamma}
$$

Then, we calculate the potential $\boldsymbol{P}^{h} \xi_{\gamma}^{\prime}$ for the density $\xi_{\gamma}^{\prime}=\boldsymbol{P}_{\gamma}^{h} \xi_{\gamma}$ and interpolate it (operation $\boldsymbol{R}_{\sigma_{1}}$ ) from $\mathrm{N}_{e x}$ to the nodes $\sigma_{1} \subset \Gamma_{1}$ :

$$
\left.\boldsymbol{u}\right|_{\sigma_{1}}=\boldsymbol{R}_{\sigma_{1}} \boldsymbol{P}^{h} \xi_{\gamma}^{\prime} \equiv \boldsymbol{R}_{\sigma_{1}} \boldsymbol{P}^{h} \xi_{\gamma}
$$

The second equality in (2.49) holds because of the projection property of $\boldsymbol{P}_{\gamma}^{h}$. Finally, the missing values of the solution at the nodes $\Gamma_{1}$ are obtained from $\left.\boldsymbol{u}\right|_{\sigma_{1}}$ by means of interpolation along the surface $\Gamma_{1}$, which altogether yields the nonlocal DPM-based ABC's in the form

$$
\left.\boldsymbol{u}\right|_{\Gamma_{1}}=\left.\boldsymbol{T}\left(\boldsymbol{u}, \frac{\partial \boldsymbol{u}}{\partial n}\right)\right|_{\Gamma}
$$

here the operation $\boldsymbol{T}$ is composed of the operations (2.48), (2.49), and interpolation along $\Gamma_{1}$. As mentioned above, in the course of the iteration procedure boundary condition (2.50) is applied every time we need to update the values of the solution at the ghost nodes $\Gamma_{1}$. The implementation of ABC's (2.50) can either be direct or involve preliminary calculation of the matrix $\boldsymbol{T}$. In the latter case, the runtime implementation of the ABC's (2.50) is reduced to a matrix-vector multiplication. Moreover, in this case we can do the first clustering $\boldsymbol{R}_{\sigma}$ and the last interpolation along $\Gamma_{1}$ separately, i.e., leave these operations out of the structure of $\boldsymbol{T}$. Then, instead of (2.50) one can write

$$
\left.\boldsymbol{u}\right|_{\sigma_{1}}=\boldsymbol{T}^{\prime} \xi_{\sigma}
$$

where both the dimension of $\boldsymbol{T}^{\prime}$ and its computational cost are many times smaller than those of $T$ from (2.50).

Let us also note that we need to know the potential only on some neighborhood of the surface $\Gamma_{1}$ (see (2.49)). At the same time, according to (2.45) and (2.46) the density of the potential differs from zero only near $\gamma$. Therefore, for both direct $T^{(y)}, T^{(z)}$ (see $(2.29)$ ) and inverse $\tilde{T}^{(y)}, \tilde{T}^{(z)}$ (see (2.30)) transforms we actually have to take into account only a few "non-zero" nodes out of the total numbers of $2 J_{y}+1$ and $J_{z}+1$ along $y$ and $z$, respectively. This effectively makes the computational cost of these transforms to grow linearly rather than quadratically with respect to $2 J_{y}+1$ and $J_{z}+1$, and obviously implies a very substantial reduction of the required computer resources. 


\section{Numerical Results.}

3.1. Two-Dimensional Summary. For the reason of completeness, we first briefly comment on the two-dimensional results from our previous work (see $[14,15,18]$ ). In that work, we have calculated the subsonic and transonic viscous flows past single-element airfoils (NACA0012 and RAE2822).

The two-dimensional computational domain is formed by the C-type curvilinear grid generated around the airfoil. On this grid, the Navier-Stokes equations are integrated using the code FLOMG by Swanson and Turkel $[10,11,12]$. The standard treatment of the external boundary in the code FLOMG is based on the locally one-dimensional characteristics analysis, which may or may not be supplemented by the point-vortex correction [32].

Basic conclusions that could be drawn from our two-dimensional numerical experience are the following. The DPM-based $\mathrm{ABC}$ 's are geometrically universal, algorithmically simple and easy to implement along with the existing solver. For the large computational domains (30-50 chords of the airfoil), the performance of the standard methods and the DPM-based ABC's is very close to one another. As, however, the artificial boundary approaches the airfoil the discrepancy between the corresponding solutions increases. The lift and drag coefficients obtained on the basis of the twodimensional version of boundary conditions (2.50) deviate from their asymptotic ( 50 chords) values much slighter (within fractions of one percent) than the coefficients obtained with the local ABC's do. In other words, the nonlocal DPM-based ABC's allow one to use much smaller computational domains (as small as 2-3 chords) than the standard boundary conditions do and to still maintain high accuracy of of the numerical solution. Moreover, if we compare three models: DPM-based, point-vortex, and standard local (characteristics-based), then it turns out that the DPM-based ABC's display the best performance for small computational domains, the performance of the local characteristic boundary conditions for small domains is very poor, and the point-vortex boundary conditions perform much better for the lift than they do for the drag coefficient. This behavior seems reasonable since the point-vortex model is a lift-based treatment.

We also note that for certain variants of computation the DPM-based ABC's may noticeably speed up (by up to a factor of three) the convergence of the multigrid iterations, see $[13,14,15]$. Some discussion on combined implementation of the DPM-based ABC's with multigrid is contained in $[18]$.

3.2. Three-Dimensional Computations. The DPM-based boundary conditions (2.50) have been combined with the interior Navier-Stokes solver and used for calculating viscous flows around the ONERA M6 wing for different regimes that range from very low to transonic Mach numbers and include both attached and separated turbulent flows.

We use the NASA-developed code TLNS3D by Vatsa, et al. [21] to integrate the thin-layer equations on the curvilinear $\mathrm{C}-\mathrm{O}$ grid (see Figures 2.1 and 2.2) generated around the wing. The code is based on the central-difference finite-volume discretization in space with the first- and third-order artificial dissipation. The steady-state solution is obtained by means of a pseudo-time iteration procedure; the integration in time is done by the five-stage Runge-Kutta algorithm (with the Courant number calculated locally) supplemented by the residual smoothing. For the purpose of accelerating the convergence, the multigrid methodology is implemented; in our computations we used three subsequent grid levels with V cycles; the full multigrid methodology (FMG) could be employed as well. In addition, we use the preconditioning technique of [49] to improve the convergence to steady state. We implement the DPM-based ABC's (2.50) only on the finest level of multigrid on the final FMG stage; the boundary data for coarser levels are provided by the coarsening procedure. Moreover, even on the finest level we implement the DPM-based ABC's only on the first and the last Runge-Kutta stages, which has been shown to make very little difference compared to the implementation on all five stages; the boundary data for the three intermediate 
stages are provided from the DPM-based ABC's on the first stage. Unlike the two-dimensional case, the standard treatment of the external boundary in three dimensions (code TLNS3D) is based merely on the locally one-dimensional characteristics analysis and extrapolation (as the point-vortex model is not applicable).

All three-dimensional flows that we have analyzed are turbulent. In the near field (i.e., inside $D_{i n}$ ), the Navier-Stokes solver is supplemented by a special turbulence model to account for the corresponding phenomena. Depending on the specific flow variant, either an algebraic or a differential turbulence model can be employed. In the far field, we use Boussinesq's concept of the effective turbulent viscosity, i.e., effective Reynolds number (see [15]). This simplest approach has been found to produce accurate results when incorporated in the structure of the DPM-based ABC's. The value of the Prandtl number for all the calculations was either $\operatorname{Pr}=0.72$ (air) or $\operatorname{Pr}=1$.

In all the cases below, the auxiliary Cartesian grids are stretched along the coordinates $y$ and $z$. The stretching typically starts outside $\Gamma_{1}$; the stretching factors (we use the geometric progression) vary between 1.07 and 1.1 for different variants. The typical values of $Y$ and $Z$ that we have used also vary between 20 and 30 sizes of $D_{i n}$ in the cross-stream direction and 4 and 10 sizes of $D_{i n}$ in the span-wise direction. The uniform Cartesian grid in the vicinity of $D_{i n}$ is always chosen so that the distance between $\Gamma$ and $\Gamma_{1}$ is well resolved.

We should also emphasize that in spite of their nonlocal nature the DPM-based ABC's (2.50) are geometrically universal. In other words, these boundary conditions can be obtained for the boundary $\Gamma$ of any irregular shape by means of the same computational procedure. This conclusion directly follows from the previous considerations and has also been repeatedly corroborated in the numerical experiments. Moreover, ABC's (2.50) appear easy to incorporate in the structure of the existing flow solvers, which has been corroborated in practice as well, and which is very important from the standpoint of applications. The issue of the computational cost of boundary conditions (2.50) and some possible ways of its reduction will be addressed later on, in Section 3.2.4.

3.2.1. Low Mach Number Regime. We first consider a very low speed flow, $M_{0}=0.01$, which, in fact, is close to the truly incompressible case. Preconditioning [49] makes the analysis of this flow possible with TLNS3D. The flow is turbulent with the molecular Reynolds number based on the root chord of the wing $R e_{0}=11.7 \cdot 10^{6}$; the angle of attack is $\alpha=3.06^{\circ}$; there is no separation and the turbulence inside $D_{i n}$ is simulated using the Baldwin-Lomax algebraic model, which is based on the concept of mixing length.

Since the free-stream Mach number is so small, we have implemented here the incompressible version of the nonlocal DPM-based ABC's (2.50) constructed on the basis of matrices (2.1b). In Table 3.1, we present the results of calculations for two different computational domains of the "average radii" of 10 and 1.25 root chords of the wing, respectively (root chord means the chord length at $z=0$ ).

TABLE 3.1

ONERA M6: $M_{0}=0.01 ; R e_{0}=11.7 \cdot 10^{6} ; \alpha=3.06^{\circ}$.

\begin{tabular}{|l|c|c|c|c|}
\hline "Average radius" of $D_{i n}$ & \multicolumn{3}{|c|}{1.25 root chords } & \multicolumn{2}{|c|}{10 root chords } \\
\hline Dimension of the grid & \multicolumn{4}{|c|}{$197 \times 49 \times 33$} \\
\hline Type of ABC's & standard & DPM & standard & DPM \\
\hline \hline Full lift, $C_{L}$ & 0.2052 & 0.1954 & 0.1940 & 0.1939 \\
\hline Relative error & $5.78 \%$ & $0.77 \%$ & $0 \%$ & $0 \%$ \\
\hline \hline Full drag, $C_{D} \times 100$ & 0.695 & 0.685 & 0.681 & 0.681 \\
\hline Relative error & $2.1 \%$ & $0.58 \%$ & $0 \%$ & $0 \%$ \\
\hline
\end{tabular}

In both cases, we used the C-O type grids of the same dimension $197 \times 49 \times 33$; for the small 
domain the grid was obtained by scaling down the bigger grid and was obviously finer in the near field. One can see that for the big domain the results (force coefficients $C_{L}$ and $C_{D}$ ) obtained using both methods are very close to each other. However, as the domain shrinks the accuracy obtained with the DPM-based procedure appears much better than the accuracy provided by the standard methodology. In other words, the nonlocal DPM-based ABC's (2.50) allow one to essentially reduce the size of the computational domain without compromising the accuracy. This confirms that if the structure of the far-field solution is correctly taken into account by means of the ABC's then within a certain range of domain sizes the computed near-field solution becomes essentially domainindependent. We also note that as the near-field grid for the small domain is finer than for the big domain then the associated truncation error can be expected smaller.

3.2.2. Subsonic Regime. The next case is a subcritical (i.e., fully subsonic) compressible flow for $M_{0}=0.5$. Here, the free-stream Mach number is already high enough to make the compressibility effects very essential but on the other hand, it is still not too high so that the flow remains locally subsonic throughout the entire domain. The angle of attack and the molecular Reynolds number for this case are the same as for the previous one: $\alpha=3.06^{\circ}, R e_{0}=11.7 \cdot 10^{6}$. The flow is also fully attached and the turbulence model inside $D_{i n}$ is algebraic (Baldwin-Lomax).

The DPM-based ABC's (2.50) for this case were constructed on the basis of non-symmetrized matrices $(2.1 \mathrm{c})$. For this specific value of Mach number, $M_{0}=0.5$, the "extent of non-symmetry" in the system matrices (2.1c) still appears quite acceptable. However, for low Mach numbers $M_{0} \lesssim 0.1$ treated in the compressible framework (unlike in Section 3.2.1), the usage of symmetrizer (2.3) and matrices (2.4) can be recommended. On the other hand, we should note that in work [18] we have been able to obtain accurate two-dimensional results for $M_{0}=0.01$ without symmetrizing the system matrices in boundary conditions.

In Table 3.2, we compare the results of calculations for three different computational domains.

TABLE 3.2

ONERA M6: $M_{0}=0.5 ; R e_{0}=11.7 \cdot 10^{\complement} ; \alpha=3.06^{\circ}$.

\begin{tabular}{|c|c|c|c|c|c|c|}
\hline "Average radius" of $D_{\text {in }}$ & $1.25 \mathrm{root}$ & chords & 2 root & lords & 10 root & hords \\
\hline Dimension of the grid & \multicolumn{6}{|c|}{$197 \times 49 \times 33$} \\
\hline Type of ABC's & standard & DPM & standard & DPM & standard & DPM \\
\hline Full lift, $C_{L}$ & 0.2218 & 0.2065 & 0.2185 & 0.2065 & 0.2081 & 0.2072 \\
\hline Relative error & $6.58 \%$ & $0.34 \%$ & $5.0 \%$ & $0.34 \%$ & $0 \%$ & $0 \%$ \\
\hline Full drag, $C_{D} \times 100$ & 0.817 & 0.791 & 0.793 & 0.791 & 0.787 & 0.788 \\
\hline Relative error & $3.8 \%$ & $0.38 \%$ & $0.76 \%$ & $0.38 \%$ & $0 \%$ & $0 \%$ \\
\hline
\end{tabular}

Like in the previous case (Section 3.2.1), here the DPM-based ABC's produce much more accurate solutions on the small computational domains than standard boundary conditions do. This obviously amounts to either saving the computer resources while preserving the accuracy of computations or improving the accuracy while keeping the computational cost at the same level. Of course, lower levels of the truncation error for finer grids on the small domains can also be anticipated here as in the foregoing low Mach number case.

3.2.3. Transonic Regime. Most of the standard test cases for flows around the ONERA M6 wing are transonic (see, e.g., the experimental work [50]). In such flows the free-stream Mach number is sufficiently high so that the local speed exceeds the speed of sound in some bounded region near the upper surface of the wing. This leads to the formation of a supersonic (i.e., supercritical) "bubble", which typically has a sonic-surface type upstream boundary and a shockwave type downstream boundary. 
Attached Flow. The first transonic case that we present is $M_{0}=0.84, \alpha=3.06^{\circ}, R e_{0}=$ $11.7 \cdot 10^{6}$. In this case, the angle of attack $\alpha$ remains sufficiently small so that the weak shock $\because$ the upper surface of the wing does not cause the flow separation. Therefore, we still use the Baldwin-Lomax model for simulating the turbulence inside $D_{i n}$. An important difference compared to the previously studied cases is that here we cannot bring the artificial boundary as close to the wing as done in Sections 3.2.1 and 3.2.2. The reason is that our far-field treatment is purely subsonic and therefore, the artificial boundary should not come to the boundary of the supercritical butisle too close. Therefore, we ran our computations for two domains, the "radius" of the big one is still about 10 root chords of the wing and the "radius" of the small one is about 3 root chords of the wing. Unlike in the previous cases, here we constructed the $\mathrm{C}-\mathrm{O}$ grids of different dimension for the domains of different size; the smaller ( 3 root chords) grid is an exact subset of the bigger (10 root chords) grid. This has been done in order to completely eliminate any influence that the change of the grid in the near field may possibly exert on the calculated solution.

The nonlocal ABC's (2.50) for this case were again constructed on the basis of matrices (2.1c). In Table 3.3, we compare the computed results (calculated lift $C_{L}$ and $\operatorname{drag} C_{D}$ coefficients) for two different types of ABC's on two different domains.

TABLE 3.3

ONERA M6: $M_{0}=0.84 ; R e_{0}=11.7 \cdot 10^{6} ; \alpha=3.06^{\circ}$.

\begin{tabular}{|l|c|c|c|c|}
\hline "Average radius" of $D_{i n}$ & \multicolumn{2}{|c|}{3 root chords } & \multicolumn{2}{c|}{10 root chords } \\
\hline Dimension of the grid & \multicolumn{2}{|c|}{$197 \times 49 \times 33$} & $209 \times 57 \times 33$ \\
\hline Type of ABC's & standard & DPM & standard & DPM \\
\hline \hline Full lift, $C_{L}$ & $0.298 \pm 0.004$ & 0.2798 & 0.2805 & 0.2786 \\
\hline Relative error & $6.24 \% \pm 1.43 \%$ & $0.43 \%$ & $0 \%$ & $0 \%$ \\
\hline \hline Full drag, $C_{D} \times 10$ & $0.168 \pm 0.008$ & 0.1537 & 0.1542 & 0.1531 \\
\hline Relative error & $8.95 \% \pm 5.19 \%$ & $0.39 \%$ & $0 \%$ & $0 \%$ \\
\hline
\end{tabular}

For the small computational domain, the DPM-based ABC's again clearly outperform the standard method from the standpoint of accuracy. We also note that in this case the total number of nodes in the bigger grid is about $25 \%$ more than in the smaller grid, which obviously implies an accordant increase of the associated cost of computations.

Even more important, for the transonic case the DPM-based ABC's influence not only the final accuracy of the solution but also the convergence rate of the iteration procedure employed inside $D_{i n}$. Namely, for the standard ABC's the multigrid iterations on the small computational domain converge noticeably slower than they do for the DPM-based ABC's. In fact, for the same $500 \mathrm{~V}$ cycles on the finest multigrid level, we simply have not been able to obtain a stable solution for the 3 root chords domain with the standard boundary conditions. That's why the corresponding data in Table 3.3 are given with the error bands indicated. The convergence history for the transonic computations on the 3 chords domain is given in Figure 3.1a for the residual of the continuity equation and in Figure 3.1b for the number of supersonic points in the domain. Note, the latter quantity is deemed very sensitive for calculation of the transonic flows. 


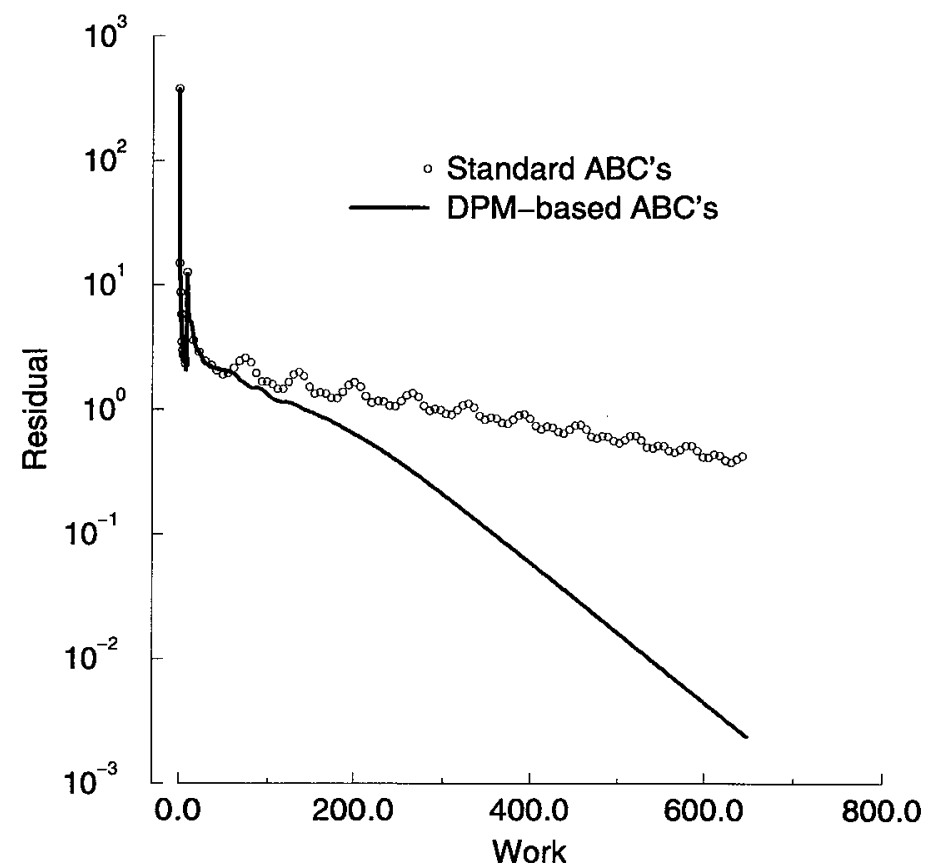

FIG. 3.1A. ONERA M6: $M_{0}=0.84, R e_{0}=11.7 \cdot 10^{6}, \alpha=3.06^{\circ}$. Convergence history for the residual of the continuity equation. "Average radius" of $D_{i n}$ is 3 root chords of the wing; the dimension of the grid is $197 \times 49 \times 33$.

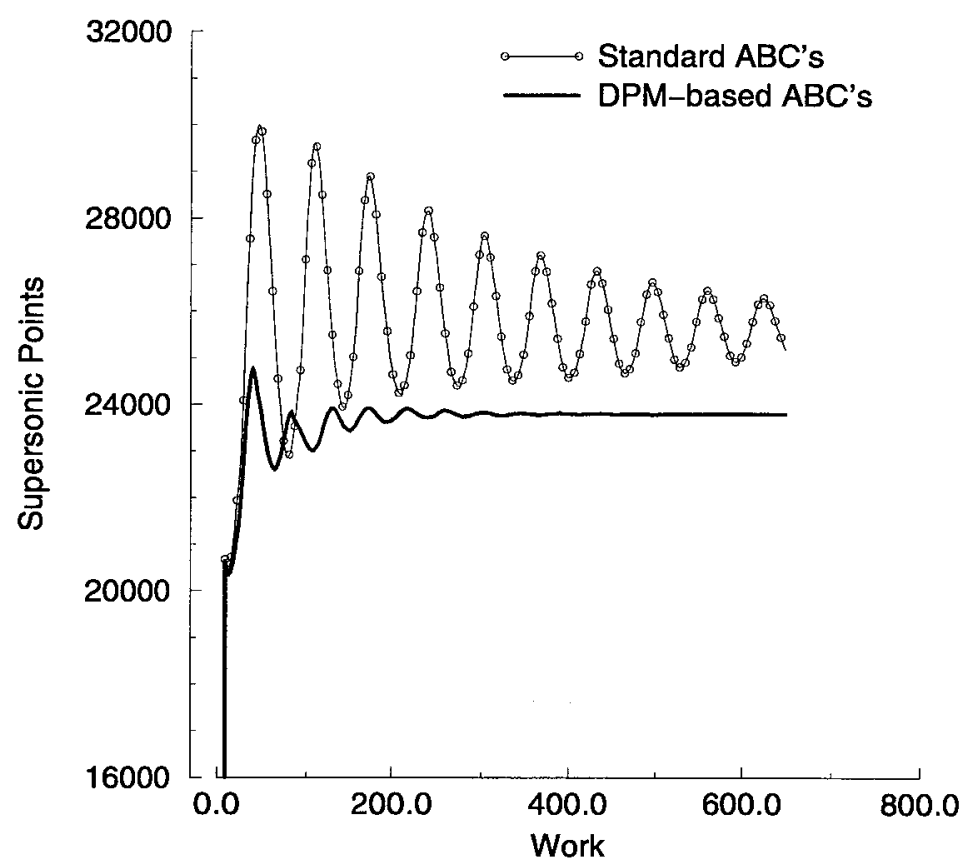

FIG. 3.1B. ONERA M6: $M_{0}=0.84, R e_{0}=11.7 \cdot 10^{\epsilon}, \alpha=3.06^{\circ}$. Convergence history for the number of supersonic nodes in the domain. "Average radius" of $D_{i n}$ is 3 root chords of the wing; the dimension of the grid is $197 \times 49 \times 33$.

From Figures 3.1 one can easily see that the difference in the multigrid convergence rates for the different types of ABC's can be as big as approximately a factor of three.

The history of convergence of the same two quantities for the big (10 root chords) computational 
domain is presented in Figures 3.2.

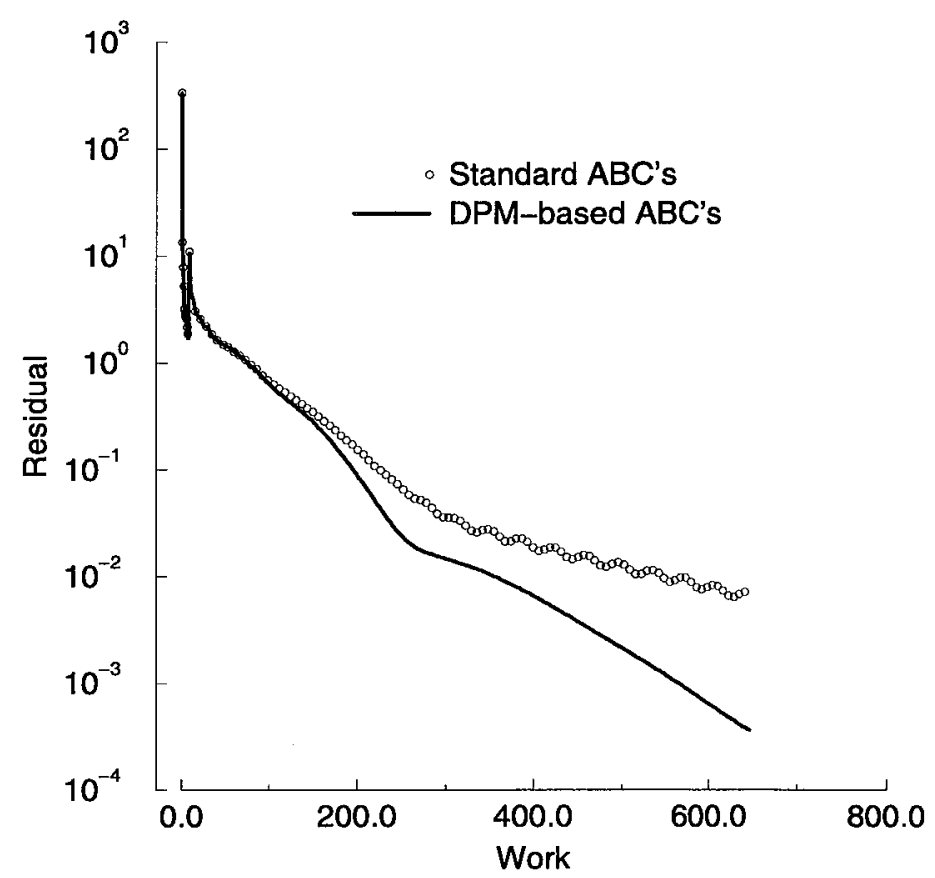

FIG. 3.2A. ONERA M6: $M_{0}=0.84, R e_{0}=11.7 \cdot 10^{\epsilon}, \alpha=3.06^{\circ}$. Convergence history for the residual of the continuity equation. "Average radius" of $D_{i n}$ is 10 root chords of the wing; the dimension of the grid is $209 \times 57 \times 33$.

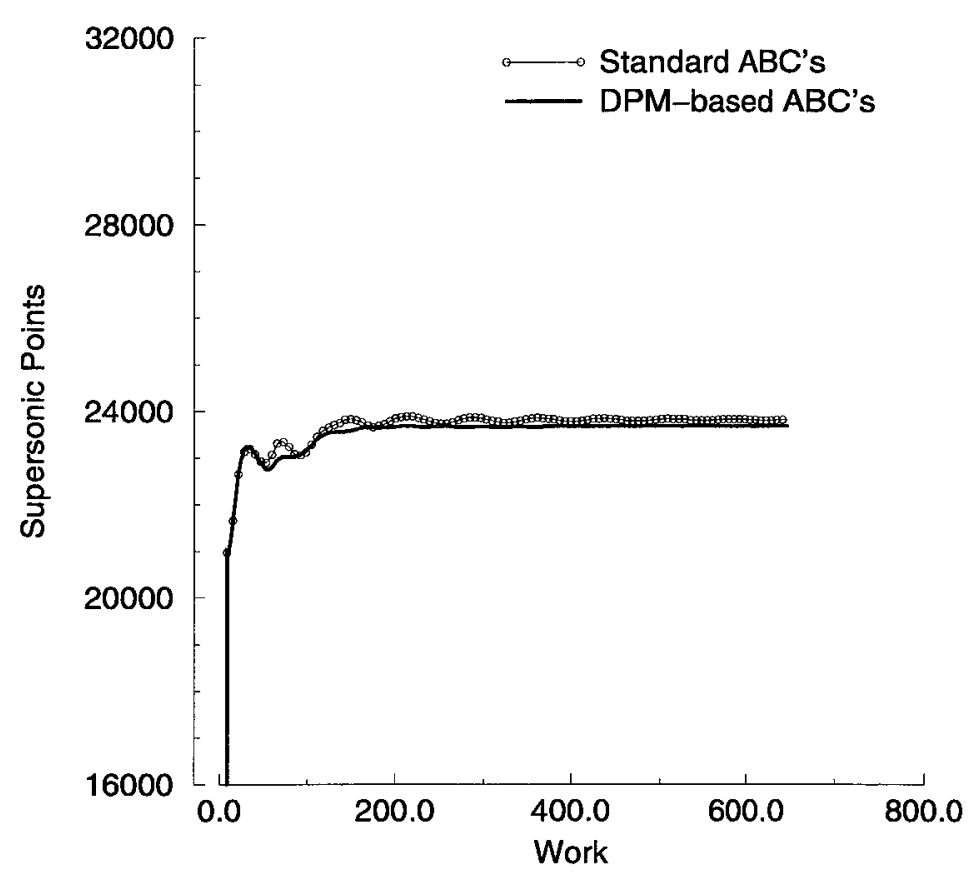

FIG. 3.2B. ONERA M6: $M_{0}=0.84, R e_{0}=11.7 \cdot 10^{6}, \alpha=3.06^{\circ}$. Convergence history for the number of supersonic nodes in the domain. "Average radius" of $D_{i n}$ is 10 root chords of the wing; the dimension of the grid is $209 \times 57 \times 33$.

We see that that in this case the DPM-based ABC's also provide for some convergence speedup, 
although the difference between the two methodologies appears less dramatic. This seems reasonable because one could generally expect that the bigger the computational domain, the smaller is the influence that the external boundary conditions exert on the numerical procedure.

Let us also note that on the small ( 3 root chords) domain the two algorithms apparently converge to quite different solutions (this is most clearly seen in Figure $3.1 \mathrm{~b}$ ), whereas Figure $3.2 \mathrm{~b}$ allows one to assume that on the big (10 root chords) domain the final solutions are close to one another. The data from Table 3.3 corroborate these conclusions. This behavior of the solution again fits into the aforementioned concept that the overall impact of the ABC's on the computational algorithm decreases as the domain enlarges.

Separated Flow. When one increases the angle of attack $\alpha$ in the transonic regime, the flow pattern changes. The shock on the upper surface of the wing becomes stronger. Since the chord length of the wing decreases span-wise as $z$ increases (see Figure 2.1), then the stream-wise size of the supersonic bubble decreases as well, and eventually the upstream sonic surface and the downstream shock wave meet somewhere in the area close to the wingtip. For sufficiently strong shocks this, in particular, produces flow separation on the upper surface of the wing. We have analyzed the separated flow of this type for $M_{0}=0.84, \alpha=5.06^{\circ}, R e_{0}=11.7 \cdot 10^{6}$.

The separation zone on the upper surface of the wing for this case is relatively small, the flow fully re-attaches before the trailing edge so that no phenomena associated with the separation are present in the wake. However, the simulation of such flows already requires more sophisticated turbulence models inside the computational domain; we have used the the two-equation Menter's model [51]. Moreover, it requires much finer grids in the near field than the simulation of the attached flows does.

As in the previous transonic case, the global ABC's (2.50) are constructed here on the basis of matrices (2.1c). The computations are conducted for two different domains of the "average radii" of 3 and 10 root chords of the wing, respectively, on the grids of the same dimension $193 \times 49 \times 33$; the smaller grid is obtained by scaling down the bigger grid (analogously to Sections 3.2 .1 and 3.2.2). In Figure 3.3, we present the distribution of the pressure coefficient

$$
C_{p}=\frac{p-p_{0}}{\frac{1}{2} \rho_{0} u_{0}^{2}}
$$

on the upper and lower surfaces of the wing in the cross-section $z=$ const at the $90 \%$ of semi-span. The $90 \%$ of semi-span station corresponds to the area of developed separation. The three solutions that we have computed in this case are for the global DPM-based ABC's on the 3 and 10 root chords domains and standard $\mathrm{ABC}$ 's on the 10 chords domain. These solutions are compared in Figure 3.3 against the experimental data.

From Figure 3.3 we conclude that all three numerical solutions very well match one another and also match the experimental data to a reasonable degree of accuracy. We also emphasize that analogously to the previous cases, the DPM-based global ABC's (2.50) are quite capable of generating an accurate numerical solution on the small domain for this separated flow case. On the other hand, unlike in the previous cases, the standard ABC's are simply unable to produce a convergent solution on the 3 root chords computational domain for the $\alpha=5.06^{\circ}$ separated flow around the ONERA M6 wing. In other words, the multigrid algorithm with the standard ABC's fails to converge on the small computational domain. Let us recall that for an easier case of $\alpha=3.06^{\circ}$ the convergence of the standard procedure on the small domain was only slowed down but not completely destroyed. The history of convergence of the residual of continuity equation for the case $\alpha=5.06^{\circ}$ on the small domain is presented in Figure 3.4a. 


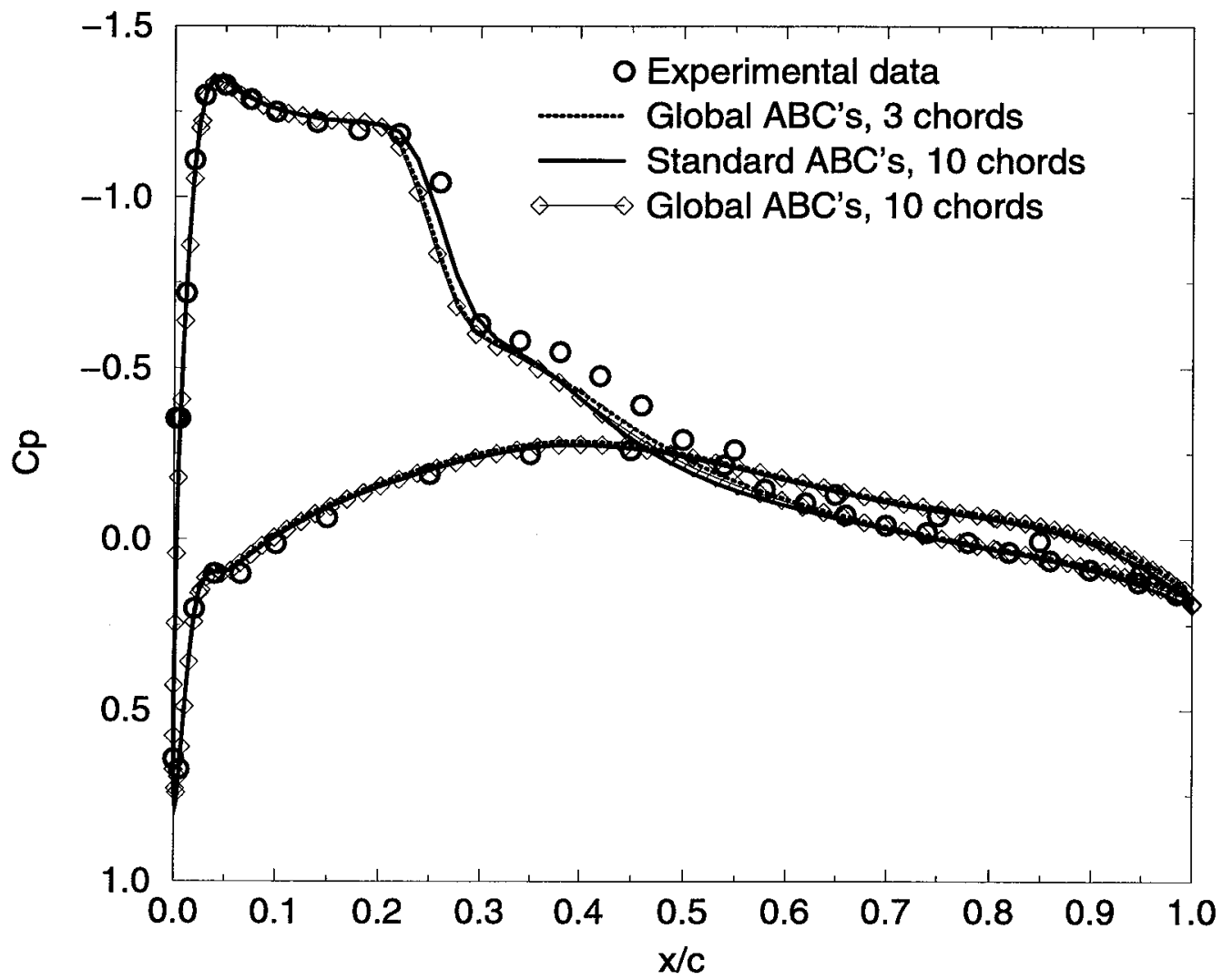

FIG. 3.3. ONERA M6: $M_{0}=0.84, R e_{0}=11.7 \cdot 10^{6}, \alpha=5.06^{\circ}$. Surface pressure distribution at the $90 \%$ of semi-span ( $x / c: x$ is the coordinate calculated from the leading edge, $c$ is the local chord length). Dimension of all grids is $193 \times 49 \times 33$.

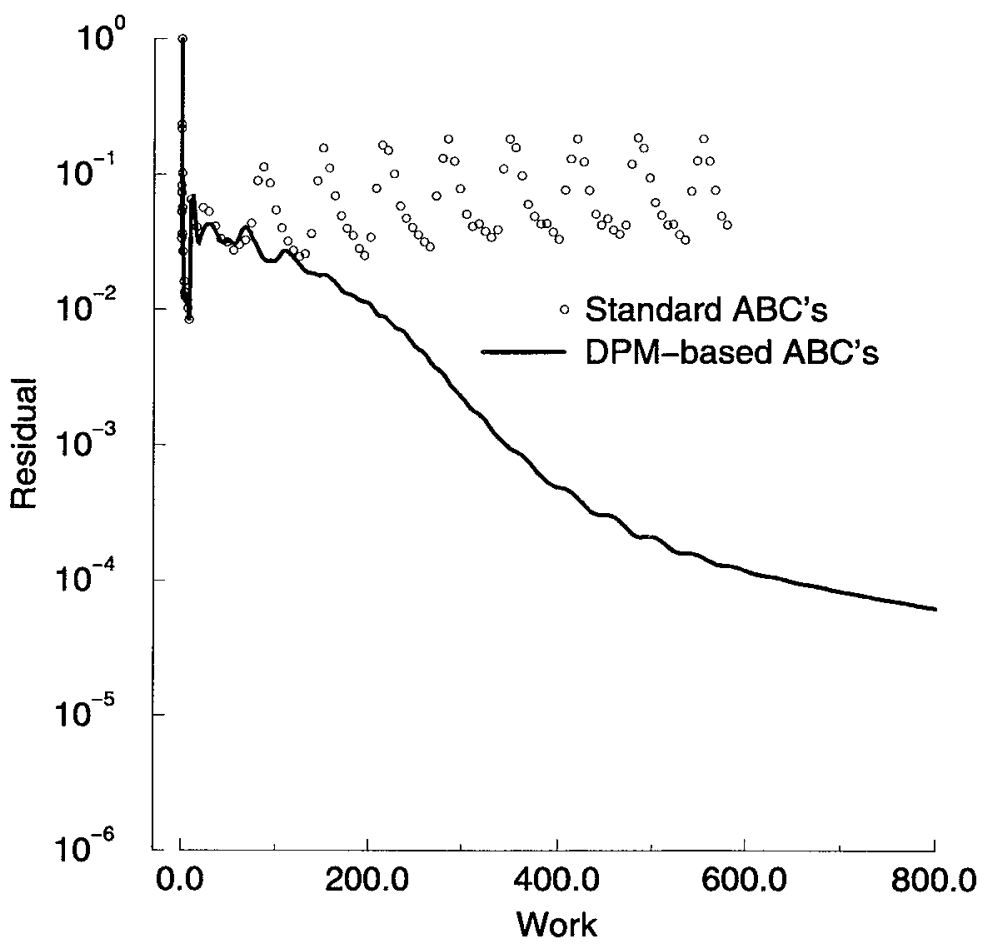

FIG. 3.4A. ONERA M6: $M_{0}=0.84, R e_{0}=11.7 \cdot 10^{6}, \alpha=5.06^{\circ}$. Convergence history for the residual of the continuity equation. "Average radius" of $D_{i n}$ is 3 root chords of the wing; the dimension of the grid is $197 \times 49 \times 33$. 
At the same time, on the big (10 root chords) domain both algorithms for the $\alpha=5.06^{\circ}$ case converge at the same rate, see Figure $3.4 \mathrm{~b}$.

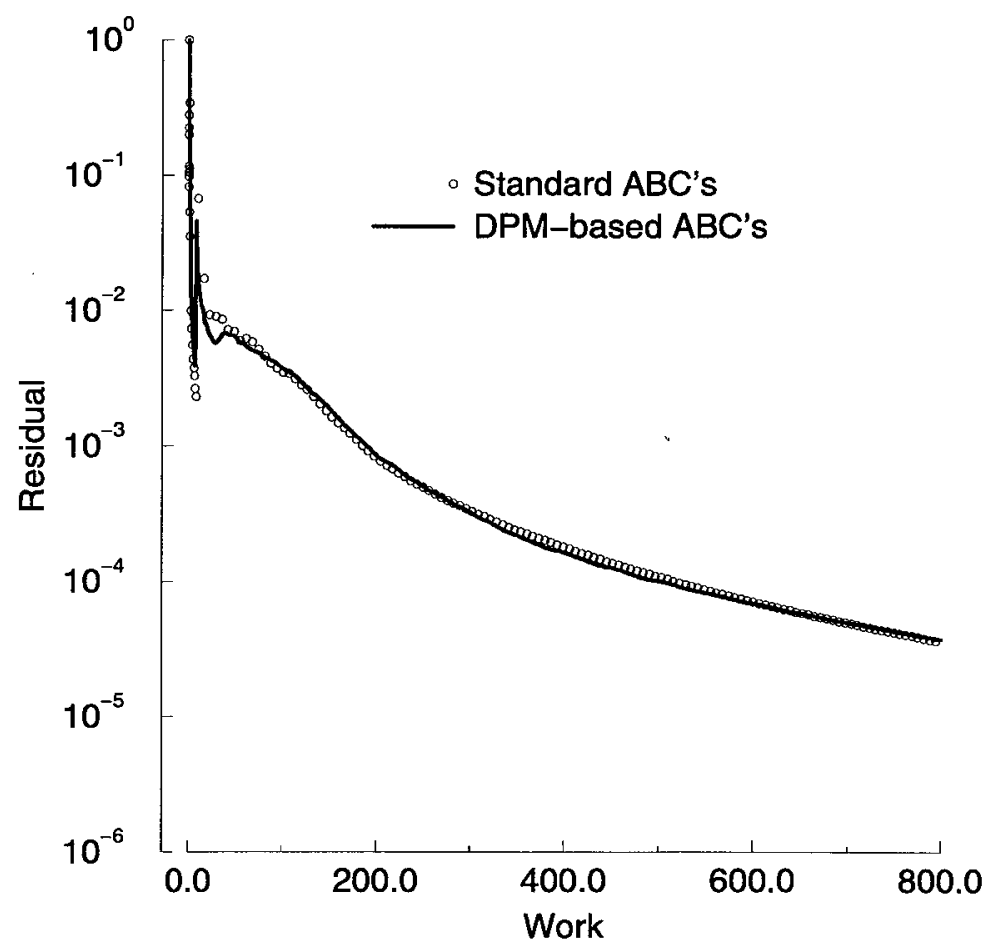

FIG. 3.4B. ONERA M6: $M_{0}=0.84, R e_{0}=11.7 \cdot 10^{6}, \alpha=5.06^{\circ}$. Convergence history for the residual of the continuity equation. "Average radius" of $D_{i n}$ is 10 root chords of the wing; the dimension of the grid is $197 \times 49 \times 33$.

Figures 3.3 and 3.4 allow us to conclude that the nonlocal DPM-based ABC's (2.50) not only speed up the convergence of the multigrid iterations but are generally capable of increasing the robustness of the entire numerical procedure.

3.2.4. Computational Cost of the DPM-based ABC's. In all the three-dimensional computations described above, the DPM-based ABC's were implemented directly, without computing the matrix of operator $\boldsymbol{T}$ from (2.50). By applying the new procedure only on the first and the last Runge-Kutta stages and only on the finest multigrid level, the total number of the required calculations of generalized potential has been brought to a minimum. In so doing, the average cost of application of the DPM-based ABC's (2.50) adds about 20-25\% of the CPU time to the cost of the same procedure with the standard (characteristics-based) boundary conditions. This extra expense is not high (taking into account the improvement of accuracy); moreover, it can often be compensated for and even noticeably prevailed over by the convergence acceleration and the reduction of the domain size. Besides, to explicitly decrease the computational cost associated with the DPM-based ABC's we plan on the future use of the entry-wise interpolation of boundary operators (see [22]) and/or the multiresolution based methodologies (see [20, 22]). We expect that the latter can also be employed when implementing the DPM-based ABC's for multi-block grids.

4. Conclusions. The new global ABC's for calculating steady-state external viscous flows in three space dimensions have been constructed on the basis of the difference potentials method. The approach generalizes and extends our previous two-dimensional results.

The new ABC's are capable of greatly reducing the size of the computational domain (compared to the standard methods) while still maintaining high accuracy of the numerical solution. This size reduction amounts to either the possibility of refining the grid in the near field, which potentially 
leads to increasing the accuracy, or usage of the smaller-dimension grids while keeping the accuracy at the same level. Moreover, the DPM-based ABC's may noticeably speed up the convergence of the multigrid iterations and generally improve the robustness of the entire numerical procedure. Finally, the new boundary conditions appear geometrically universal and easy to incorporate in the structure of the existing flow solvers. The properties of the new ABC's have been corroborated experimentally by computing the subsonic and transonic flows past the ONERA M6 wing using the NASA-developed code TLNS3D.

Acknowledgments. I am most grateful to V. N. Vatsa of NASA Langley Research Center for giving me an opportunity to use the code TLNS3D and for his diverse and extremely valuable help on every stage of this work. I am also very thankful to E. B. Parlette of Vigyan, Inc., for generating the grids used in the foregoing computations. Numerous discussions on the subject with R. C. Swanson and J. L. Thomas of NASA Langley Research Center have undoubtedly contributed much to the improvement of this paper.

\section{REFERENCES}

[1] S. V. Tsynkov, Artificial Boundary Conditions Based on the Difference Potentials Method, NASA Technical Memorandum No. 110265, Langley Research Center, July 1996.

[2] D. Givoli, Non-reflecting Boundary Conditions, J. Comput. Phys., 94 (1991) pp. 1-29.

[3] D. Grvol, Numerical Methods for Problems in Infinite Domains, Elsevier, Amsterdam, 1992.

[4] A. P. Calderon, Boundary-Value Problems for Elliptic Equations, in Proceedings of the Soviet-American Conference on Partial Differential Equations at Novosibirsk, Fizmatgiz, Moscow, 1963, pp. 303-304.

[5] R. T. SeELEY, Singular Integrals and Boundary Value Problems, Amer. J. Math., 88 (1966) pp. 781-809.

[6] V. S. Ryaben'KII, Boundary Equations with Projections, Russian Math. Surveys, 40 (1985) pp. 147-183.

[7] V. S. Ryaben'Kin, Difference Potentials Method for Some Problems of Continuous Media Mechanics, Nauka, Moscow, 1987. (in Russian)

[8] V. S. Ryaben'KII, Difference Potentials Method and its Applications, Math. Nachr., 177 (1996) pp. 251-264.

[9] S. G. Mikhlin, N. F. Morozov, and M. V. Paukshto, The Integral Equations of the Theory of Elasticity, B. G. Teubner Verlagsgesellschaft, Stuttgart, 1995.

[10] R. C. Swanson AND E. TuRKel, A Multistage Time-Stepping Scheme for the Navier-Stokes Equations, AIAA Paper No. 85-0035, AIAA 23rd Aerospace Sciences Meeting, Reno, Nevada, 1985.

[11] R. C. Swanson and E. Turkel, Artificial Dissipation and Central Difference Schemes for the Euler and Navier-Stokes Equations, AIAA paper No. 87-1107-CP, AIAA 8th Computational Fluid Dynamics Conference, Honolulu, Hawaii, 1987.

[12] R. C. SWANSON AND E. TURKEL, Multistage Schemes with Multigrid for the Euler and Navier-Stokes Equations. Volume I: Components and Analysis, NASA Technical Paper No. 3631, Langley Research Center, 1997.

[13] V. S. Ryaben'KII AND S. V. Tsynkov, Artificial Boundary Conditions for the Numerical Solution of External Viscous Flow Problems, SIAM J. Numer. Anal., 32 (1995) pp. 1355-1389.

[14] S. V. Tsynkov, An Application of Nonlocal External Conditions to Viscous Flow Computations, J. Comput. Phys., 116 (1995) pp. 212-225.

[15] S. V. Tsynkov, E. Turkel, and S. Abarbanel, External Flow Computations Using Global Boundary Conditions, AIAA J., 34 (1996) pp. 700-706; also AIAA Paper No. 95-0562, 33rd AIAA Aerospace Sciences Meeting and Exhibit, Reno, NV, January 1995.

[16] V. S. Ryaben'Kil AND S. V. Tsynkov, An Effective Numerical Technique for Solving a Special Class of Ordinary Difference Equations, Appl. Numer. Math., 18 (1995) pp. 489-501.

[17] S. V. Tsynkov, Artificial Boundary Conditions for Computation of Oscillating External Flows, to appear in SIAM J. Sci. Comput.; also NASA Technical Memorandum No. 4714, Langley Research Center, August 1996.

[18] V. S. Ryaben'Kil and S. V. Tsynkov, An Application of the Difference Potentials Method to Solving External Problems in CFD, NASA Technical Memorandum No. 110338, Langley Research Center, March 1997; also to appear in CFD Review 1996, M. Hafez and K. Oshima, eds.

[19] S. V. Tsynkov, Nonlocal Artificial Boundary Conditions for Computation of External Viscous Flows, in: Computational Fluid Dynamics'96, Proceedings of the Third ECCOMAS CFD Conference, September 9-13, 1996, Paris, France, J.-A. Desideri, C. Hirsch, P. Le Tallec, M. Pandolfi, and J. Périaux, eds., John Wiley \& Sons, 1996, pp. 512-518.

[20] S. V. Tsynkov, Artificial Boundary Conditions for Infinite-Domain Problems, to appear in Proceedings of the ICASE/LaRC Workshop on Barriers and Challenges in Computational Fluid Dynamics, Hampton, August 5-7, 1996, M. D. Salas et al., eds., Kluwer Academic Publishers, 1997. 
[21] V. N. Vatsa, M. D. Sanetrik, AND E. B. Parlette, Development of a Flexible and Efficient Multigrid-Based Multiblock Flow Solver, AIAA Paper No. 93-0677, 31st AIAA Aerospace Sciences Meeting and Exhibit, Reno, NV, January 1993.

[22] S. V. Tsynkov and V. N. Vatsa, An Improved Treatment of External Boundary for Three-Dimensional Flow Computations, submitted to the 13th AIAA Computational Fluid Dynamics Conference, June 1997.

[23] D. Anderson, J. Tannehill, and R. Pletcher, Computational Fluid Mechanics and Heat Transfer, Hemisphere Publishing Corporation, New York, 1984.

[24] S. Abarbanel and D. GotTlieb, Optimal Time Splitting for Two- and Three-Dimensional Navier-Stokes Equations with Mixed Derivatives, J. Comput. Phys., 41 (1981) pp. 1-33.

[25] S. Abarbanel, P. Duth, AND D. GotTlueb, Splitting Methods for Low Mach Number Euler and Navier-Stokes Equations, Computers \& Fluids, 17 (1989) pp. 1-12.

[26] J. Guerra And B. Gustafsson, A Numerical Method for Incompressible and Compressible Flow Problems with Smooth Solutions, J. Comput. Phys., 63 (1986) pp. 377-397.

[27] B. Gustafsson AND H. STOOR, Navier-Stokes Equations for Almost Incompressible Flow, SIAM J. Numer. Anal., 28 (1991) pp. 1523-1547.

[28] J.-Y. SA AND K. S. CHANG, Far-Field Stream Function Condition for Two-Dimensional Incompressible Flows, J. Comput. Phys., 91 (1990) pp. 398-412.

[29] R. H. Burkhart, Asymptotic Expansion of the Free-Space Green's Function for the Discrete 3-D Poisson Equation, to appear in SIAM J. Sci. Comput.

[30] R. H. Burkhart, J. Bussoletti, F. T. Johnson, S. S. Samant, and D. P. Young, Solution of the Discrete Free-Space 3-D Poisson Equation, Boeing Computer Services Technical Report, BCSTECH-94-015, April 1994.

[31] F. W. Wubs, J. W. Boerstoel, A. J. VAN DeR WeEs, Grid Size Reduction in Flow Calculations on Infinite Domains by Higher-Order Far-Field Asymptotics in Numerical Boundary Conditions, J. Engrg. Math., 18 (1984) pp. 157-177.

[32] J. L. Thomas AND M. D. Salas, Far-Field Boundary Conditions for Transonic Lifting Solutions to the Euler Equations, AIAA Paper No. 85-0020, 1985.

[33] A. Bayliss And E. Turkel, Radiation Boundary Conditions for Wave-Like Equations, Commun. Pure Appl. Math., 33 (1980) pp. 707-725.

[34] A. Bayliss And E. Turkel, Outflow Boundary Conditions for Fluid Dynamics, SIAM J. Sci. Statist. Comput. 3 (1982) pp. 250-259.

[35] A. Bayliss AND E. TuRkel, Far-Field Boundary Conditions For Compressible Flows, J. Comput. Phys., 48 (1982) pp. 182-199.

[36] A. Bayliss, M. Gunzburger, And E. Turkel, Boundary Conditions for Numerical Solution of Elliptic Equations in Exterior Domains, SIAM J. Appl. Math., 42 (1982) pp. 430-451.

[37] J. D. Cole AND L. P. CooK, Transonic Aerodynamics, Elsevier, Amsterdam, 1986.

[38] M. Drela Two-Dimensional Transonic Aerodynamic Design and Analysis Using the Euler Equations, Massachusetts Institute of Technology, Gas Turbine Laboratory Report No. 187, February 1986.

[39] M. B. Giles AND M. DrelA, Two-Dimensional Transonic Aerodynamic Design Method, AIAA Journal, 25 (1987) pp. 1199-1206.

[40] G. S. S. LUDFond, The Behavior at Infinity of the Potential Function of a Two-Dimensional Subsonic Compressible Flow, J. Math. and Phys., 30 (1951), pp. 117-130.

[41] L. D. Landau and E. M. Lifshitz, Fluid Mechanics, Pergamon Press, Oxford, 1986.

[42] H. Ashley and M. T. Landahl, Aerodynamics of Wings and Bodies, Addison-Wesley Publishing Company, Reading, Massachusetts, 1965.

[43] V. S. VladmiRov, Equations of Mathematical Physics, Dekker, New York, 1971.

[44] A. N. Kolmogonov AND S. V. Fomin, Elements of the Theory of Functions and Functional Analysis, Nauka, Moscow, 1981, (in Russian).

[45] H. Schlichting, Boundary Layer Theory, McGraw-Hill, New York, 1968.

[46] V. S. RYABEN'KII, Necessary and Sufficient Conditions for Good Definition of Boundary Value Problems for Systems of Ordinary Difference Equations, U.S.S.R. Comput. Math. and Math. Phys., 4 (1964) pp. $43-61$.

[47] F. R. Gantmakner, Applications of the Theory of Matrices, Interscience Publishers, New York, 1959.

[48] S. K. Godunov And V. S. RYaben'KII, Canonical Forms of Systems of Ordinary Linear Difference Equations with Constant Coefficients, U.S.S.R. Comput. Math. and Math. Phys., 3 (1963) pp. 281-295.

[49] E. Turkel, V. N. Vatsa, AND R. RAdesPiel, Preconditioning Methods for Low-Speed Flows, AIAA Paper No. 96-2460-CP, June 1996.

[50] V. Schmit AND F. ChaRPIN, Pressure Distributions on the ONERA M6 Wing at Transonic Mach Numbers, AGARD AR-138, 1979, p. B1.

[51] F. R. MENTER, Performance of Popular Turbulence Models for Attached and Separated Adverse Pressure Gradient Flows, AIAA Paper No. 91-1784, AIAA 22nd Fluid Dynamics, Plasma Dynamics \& Lasers Conference, Honolulu, Hawaï, June 1991. 


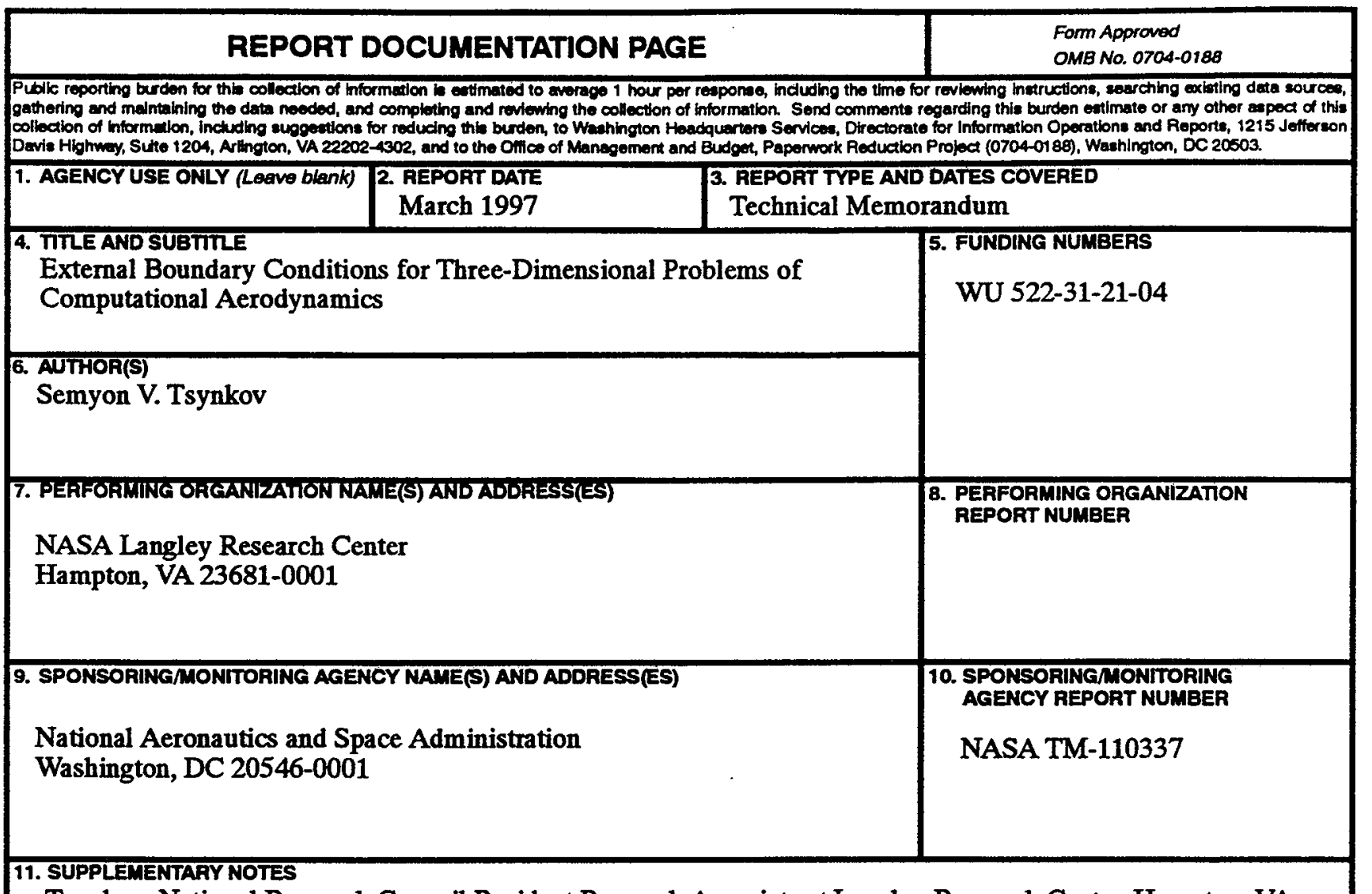

Tsynkov: National Research Council Resident Research Associate at Langley Research Center, Hampton, VA

120. DISTAIBUTION/AVAILABILTY STATEMENT

12b. DISTRIBUTION CODE

Unclassified-Unlimited

Subject Category 02

Availability: NASA CASI (301) 621-0390

\section{ABSTRACT (Maximum 200 words)}

We consider an unbounded steady-state flow of viscous fluid over a three-dimensional finite body or configuration of bodies. For the purpose of solving this flow problem numerically, we discretize the governing equations (Navier-Stokes) on a finitedifference grid. The grid obviously cannot stretch from the body up to infinity, because the number of the discrete variables in that case would not be finite. Therefore, prior to the discretization we truncate the original unbounded flow domain by introducing some artificial computational boundary at a finite distance of the body. Typically, the artificial boundary is introduced in a natural way as the external boundary of the domain covered by the grid. The flow problem formulated only on the finite computational domain rather than on the original infinite domain is clearly subdefinite unless some artificial boundary conditions (ABC's) are specified at the external computational boundary. Similarly, the discretized flow problem is subdefinite (i.e., lacks equations with respect to unknowns) unless a special closing procedure is implemented at this artificial boundary. The closing procedure in the discrete case is called the $A B C$ 's as well. In this paper, we present an innovative approach to constructing highly accurate ABC's for three-dimensional flow computations. The approach extends our previous technique developed for the two-dimensional case; it employs the finite-difference counterparts to Calderon's pseudodifferential boundary projections calculated in the framework of the difference potentials method (DPM) by Ryaben'kii. The resulting ABC's appear spatially nonlocal but particularly easy to implement along with the existing solvers. The new boundary conditions have been successfully combined with the NASA-developed production code ILIS3D and used for the analysis of wing-shaped configurations in subsonic (including incompressible limit) and transonic flow regimes. As demonstrated by the computational experiments and comparisons with the standard (local) methods, the DPM-based ABC's allow one to greatly reduce the size of the computational domain while still maintaining high accuracy of the numerical solution. Moreover, they may provide for a noticeable increase of the convergence rate of multigrid iterations.

14. SUEJEC' THAIIS

External flows; Infinite-domain problems; Artificial boundary conditions; Far-Field linearization; Boundary projections; Generalized potentials; Difference potentials method; Auxiliary problem; Separation of variables

\begin{tabular}{l|l|l|}
\hline $\begin{array}{l}\text { 17. SECUAITY CLASSIFICATION } \\
\text { OF REPOAT } \\
\text { Unclassified }\end{array}$ & $\begin{array}{c}\text { 18. SECURITY CLASSIFICATION } \\
\text { OF THIS PAGE } \\
\text { Unclassified }\end{array}$ & $\begin{array}{c}\text { 19. SECUAITY CLASSIFCATION } \\
\text { OF ABSTRACT } \\
\text { Unclassified }\end{array}$ \\
\hline
\end{tabular}

NSN 7540-01-280-5500
15. NUMBER OF PAGES 40 16. PAICE CODE A03

20. UMITATON OF ABSTRACT 